\title{
Mathematical modelling and sensitivity analysis of HIV-TB co-infection.
}

\author{
S.O. Adewale ${ }^{1}$, I.A.Olopade ${ }^{2}$, G.A. Adeniran ${ }^{3}$ and S.O, Ajao ${ }^{4}$
}

Ladoke Akintola University of Technology, (LAUTECH), Ogbomoso, P.M.B. 4000, Ogbomoso, Oyo State, Nigeria.

Ladoke Akintola University of Technology, (LAUTECH), Ogbomoso, P.M.B. 4000, Ogbomoso, Oyo State, Nigeria.

Ladoke Akintola University of Technology, (LAUTECH), Ogbomoso, P.M.B. 4000, Ogbomoso, Oyo State, Nigeria.

Ladoke Akintola University of Technology, (LAUTECH), Ogbomoso, P.M.B. 4000, Ogbomoso, Oyo State, Nigeria.

\begin{abstract}
In this paper, we formulated a new nine (9) compartmental mathematical model to have better understanding of parameters that influence the dynamical spread of Human immunodeficiency virus (HIV) interacting with Tuberculosis (TB) in a population. The model is analyzed for all the parameters responsible for the disease spread in order to find the most sensitive parameters out of all.

Sub models of HIV and TB only were considered first, followed by the full HIV-TB co-infection model. Stability of HIV model only, TB model only and full model of HIV-TB co-infection were analyzed for the existence of the disease free and endemic equilibrium points. Basic Reproduction $\operatorname{Number}\left(R_{0}\right)$ was obtained using next generation matrix method (NGM), and it has been shown that the disease free equilibrium point is locally asymptotically stable whenever $R_{0}<1$ and unstable whenever this threshold exceeds unity. i.e. $R_{0}>1$.

The relative sensitivity solutions of the model with respect to each of the parameters is calculated, Parameters are grouped into two categories: sensitive parameters and insensitive parameters.

Numerical simulation was carried out by maple software using Runge-kunta method, to show the effect of each parameter on the dynamical spread of HIV-TB co-infection, i.e. detection of infected undetected individuals plays a vital role, it decreases infected undetected individuals. Also, increased in effective contact rate has a pronounced effect on the total population; it decreases susceptible individuals and increases the infected individuals. However, effective contact rate needs to be very low in order to guaranteed disease free environment.
\end{abstract}

\section{Keywords}

Human Immunodeficiency Virus; Tuberculosis; Reproduction number; Critical points; Sensitivity analysis; Stability.

\section{Academic discipline}

Mathematics

\section{Subject classification}

Mathematics subject classification

(Method/Approach)

Bio-Mathematics

\section{COUNCIL FOR INNOVATIVE RESEARCH}

\author{
Peer Review Research Publishing System
}

\section{JOURNAL: JOURNAL OF ADVANCES IN MATHEMATICS}

VOL.11, No.8

www.cirjam.com, editoriam@gmail.com 


\section{INTRODUCTION.}

Tuberculosis (TB) is an airborne infectious disease caused by Mycobacterium tuberculosis which affects the lung and other parts of the body [3]. TB infection occurs when droplet nuclei containing tubercle bacilli are inhaled into the lungs and deposited in the alveoli. It is spread when individuals with active TB disease cough, sneeze, sing, laugh or through interaction with infectious individual. After a person becomes infected, the tuberculosis bacteria are controlled by the person's immune system and the infection becomes latent. The infection becomes active, when the bacteria spread out of control most especially when the tubercle bacilli overwhelm the immune system and break out of the tubercles in the alveoli and spread to the lungs and other parts of the body via blood stream [24]. The risk of infection with the tubercle bacilli is directly related to the degree of exposure and less to genetic or other host factors. In those with HIV, the risk of developing active TB increases to nearly $18 \%$ per year [3,5]. TB is a major global cause of disability and death, especially in developing countries. Even in many countries where its overall incidence is low, TB remains a problem; In 2006, TB caused an estimated 1.7 million deaths and 8.9 million new cases of infection [23].

The early symptoms of active Tuberculosis include: Weight loss, Fever, loss of appetite, coughing out of blood, night sweats, and back pain among others.

TB is curable disease by the implementation of antibiotics, which decrease the mortality of TB to a minimal level, for instance, a 70\% reduction in TB related mortality was recorded in the United State of America (USA) between 1945-1955 [2]. Many doctors prefer to hospitalize the patient in order to observe him or her during treatment, the method is called Direct Observed Therapy Short Course (DOTS), huge success has been achieved by this method. [1].

Human Immunodeficiency Virus (HIV) is caused by retrovirus infection; it progresses to Acquired Immunodeficiency Syndrome (AIDS) when proper treatment is not taken. HIV severely weakens immune system by attacks many blood cells, it causes havoc on the T-cell in the blood by destroying and decreasing their number, leading to decline in body's immunity to fight infection [20].. There are multiple modes of HIV transmission including Sexual intercourse, sharing needles with HIV infected persons, or via HIV-contaminated blood transfusions [21]. Infants may acquire HIV at delivery (Birth) or through breast feeding if the mother is HIV positive. This type of transmission is known as vertical transmission [6]. The term AIDS refers to only the last stage of the HIV infection after which death occurs. The HIV epidemic is sweeping the world both developed and developing nations as HIV has infected millions of people all over the world without any restriction of nationality, religion etc [18]. HIV infection in adults (75-80 percent) has been transmitted to one partner through unprotected sexual intercourse when the other partner is infected with HIV. Mother to child transmission (vertical infection accounts for more than 90 percent) of global infection to infants and children. As HIV infection progresses, immunity declines and patients tend to become more susceptible to common or even rare infections. The sexual transmission is believed globally responsible for the majority of new HIV infections [14,17]

The related spread of two or more infections has always been a cause of concern for human being. HIV-TB co-infection is the largest cause among them. Tuberculosis (TB) is the most common opportunistic disease affecting HIV positive people and the leading cause of death in patients with AIDS [8,12]. Also, the relative risk of death and development of other opportunistic infections is higher in HIV-TB co-infected patients as compared to those having only one disease out of the two. [15].

Persons co-infected with TB and HIV may spread the disease not only to other HIV-infected persons, but also members of the general population who do not participate in any of the high risk behaviours associated with HIV. [22]. In recent decades, the dramatic spread of the HIV epidemic in sub-Saharan Africa has resulted in notification rates of TB increasing up to 10 - times. The incidence of TB is also increasing in other high HIV prevalence countries where the population with HIV infection and TB overlap. [15].

Patients with latent Mycobacterium tuberculosis infection are at higher risk for progression into active TB if they are coinfected with HIV [15]. HIV significantly increases individual risk of progression to active TB in both primary TB and the reactivation of latent TB. Likewise TB fuels the progression of HIV to full blwn AIDS [10]. It is estimated that among 33.4 million people living with HIV worldwide, about one-third are also infected by MTB [11]. India has about 1.8 million new cases of tuberculosis annually. In 2012, of the estimated 1.2 million TB deaths, about one-quarter were HIV/TB co-infected patients [25]. The interaction of these deadly diseases is sweeping off the population and it is quite unfortunate that much work has not been done to model the co-infection of these deadly diseases at a population level.

Due to the threat poses by the interaction of HIV with TB, we formulated nine (9) new compartmental models to determine the parameters that influence the transmission of these deadly diseases using forward sensitivity method.

2. Mathematical model formulation. A non linear mathematical model is formulated and analyzed to study the sensitivity of parameters involved in the basic reproduction number $\left(R_{0}\right)$ on the dynamical spread of HIV- TB coinfection.

In modeling the dynamics, the total homogeneously mixing population at time $t$, denoted by $N(t)$, is divided into (9) nine mutually-exclusive compartments of Susceptible $(\mathrm{S}(\mathrm{t}))$ individuals, Latently HIV $\left(L_{H}(t)\right)$ individuals, HIV Undetected $\left(H_{U}(t)\right)$ individuals, HIV Detected $\left(H_{D}(t)\right)$ individuals, Treated HIV $\left(T_{H}(t)\right)$ individuals, Latent TB $\left(L_{T}(t)\right)$ individuals, TB Undetected $\left(T_{U}(t)\right)$ individuals, TB Detected $\left(T_{D}(t)\right)$ individuals and Recovered TB $\left(R_{T}(t)\right)$ individuals So that, 


$$
N(t)=S+L_{H}+H_{U}+H_{D}+T_{H}+L_{T}+T_{U}+T_{D}+R_{T}
$$

The Susceptible population is increased by the recruitment of individuals into the population at rate $\pi$.the population decrease by natural death rate $\mu$ and by singly infected transmission individuals. Both singly infected individuals transmit either HIV or TB infection as follows (note that we split the disease transmission process into those generated by singlyinfected infected individuals to make the formulation easier to follow).

Susceptible individuals acquire HIV infection, following effective contact with people infected with HIV only (i.e., those in the $\left(L_{H}, \eta_{U} H_{U}, \eta_{d H} H_{D}\right.$ and $\left.\eta_{T H} T_{H}\right)$ classes at a rate $\lambda_{H}$, given by

$\lambda_{H}=\beta_{H} \frac{\left(L_{H}+\eta_{U} H_{U}+\eta_{d H} H_{D}+\eta_{T H} T_{H}\right)}{N}$

Where, $\beta_{H}$ is the effective contact rate for HIV transmission.

Similarly, susceptible individuals acquire TB infection from individuals with TB only (i.e. $\left(L_{T}, \eta_{U} T_{U}, \eta_{d T} T_{D}\right.$ and $\left.\eta_{R T} R_{T}\right)$ classes at a rate $\lambda_{T}$, given by

$\lambda_{T}=\beta_{T} \frac{\left(L_{T}+\eta_{U} T_{U}+\eta_{d T} T_{D}+\eta_{R T} R_{T}\right)}{N}$

Where, $\beta_{T}$ is the effective contact rate for TB infection.

Then,

$\frac{d S}{d t}=\pi-\lambda_{H} S-\lambda_{T} S-\mu S$

A fraction $\varepsilon_{1}$ of the newly infected individuals are assumed to show no disease symptoms initially. These individuals (known as "slow progressor") are moved to the latently HIV class $\left(L_{H}\right)$. The remaining fraction, $\left(1-\varepsilon_{1}\right)$ of the newly infected individuals are assumed to immediately display disease symptoms (Fast progressors) and are moved to the undetected infectious class $H_{U}$. The population of latent class is decreased by the progression of latent HIV individual to active undetected HIV $H_{U}$ (at a rate $\kappa_{H}$ ) and also reduced by natural death rate $(\mu)$. Thus,

$\frac{d L_{H}}{d t}=\varepsilon_{1} \lambda_{H} S-\left(\kappa_{H}+\mu\right) L_{H}$

(5) The

population of undetected infected individuals is increased by the infection of Fast progressors (at the rate $\left(1-\varepsilon_{1}\right) \lambda$ ) and the development of symptoms by latently individual at the rate $\left(1-\omega_{1}\right) \kappa_{H}$ where $\omega_{1}$ is the endogenous reactivation rate. This population is decreased by natural death rate $(\mu)$ and disease induced death (at a rate $\delta_{U H}$ ) and further decreased by detection rate $\left(\gamma_{U H}\right)$ of HIV undetected infected individuals. Hence

$\frac{d H_{U}}{d t}=\left(1-\varepsilon_{1}\right) \lambda_{H} S+\left(1-\omega_{1}\right) \kappa_{H} L_{H}-\left(\gamma_{U H}+\mu+\delta_{U H}\right) H_{U}$

The population of Detected infected HIV individual increases by the fraction of latently individuals who develop disease symptoms (at the rate $\omega_{1} \kappa_{H}$ ), where $\omega_{1}$ is the endogenous reactivation rate and the detection of undetected individual at the rate $\gamma_{U H}$. The population later decreased by treatment rate $\left(\tau_{1}\right)$ for HIV detected individual and finally reduced by the natural death rate, induced mortality death rate at $\mu$ and $\delta_{U H}$ respectively. Hence we have.

$$
\frac{d H_{D}}{d t}=\omega_{1} \kappa_{H} L_{H}+\gamma_{U H} H_{U}-\left(\tau_{1}+\mu+\delta_{U H}\right) H_{D}
$$

The population of Treated HIV individuals is increased by those that have received treatment from HIV detected infected individual at the rate $\left(\tau_{1}\right)$ this population reduces by fraction of treated individual that moved back to latently HIV individuals at the rate, $(\phi)$ since treatment does not completely clears the bacteria and finally reduced by natural death rate $(\mu)$. 
Hence,

$$
\frac{d T_{H}}{d t}=\tau_{1} H_{D}-\left(\mu+\delta_{T H}\right) T_{H}
$$

A fraction $\varepsilon_{2}$ of the newly-infected individuals are assumed to show no disease symptoms initially. These individuals (Known as slow progression for TB) moved to the latently TB class $\left(L_{T}\right)$. The remaining fraction $\left(1-\varepsilon_{1}\right)$ of the newly infected individuals are assumed to immediately display disease symptoms (Fast progressor for (TB)) and are moved to the undetected infected compartment $\left(T_{U}\right)$. The population decreased by progression rate $\left(\kappa_{T}\right)$ from latent TB class to infected undetected class andnatural death rate $(\mu)$.

$$
\frac{d L_{T}}{d t}=\varepsilon_{2} \lambda_{T} S-\left(\kappa_{T}+\mu\right) L_{T}
$$

The population of undetected infectious individuals is increased by the infection of fast progressor at the rate $\left(1-\varepsilon_{1}\right) \lambda_{T}$ and the development of symptoms by latent individual at the rate $\left(1-\omega_{2}\right) \kappa_{T}$, where $\omega_{2}$ is the fraction of exposed individuals who develop symptoms and are detected. detection of undetected individual at a rate $\left(\gamma_{U H}\right)$, natural death at the rate $(\mu)$ and disease induced death at a rate $\left(\delta_{U T}\right)$. Hence, we have

$$
\frac{d T_{U}}{d t}=\left(1-\varepsilon_{2}\right) \lambda_{H} S+\left(1-\omega_{2}\right) \kappa_{T} L_{T}-\left(\gamma_{U T}+\mu+\delta_{U T}\right) T_{U}
$$

The population of detected infectious individual increases by the fraction of latent individuals who develop diseases symptoms at a rate $\omega_{2} \kappa_{T}$ and detection rate for undetected individual at the rate $\left(\gamma_{U T}\right)$. The population is decreased by those that are treated who later moved to recovered compartment at the rate $\left(\tau_{2}\right)$, natural death rate $(\mu)$ and disease induced death at a rate $\left(\delta_{d T}\right)$ This gives

$\frac{d T_{D}}{d t}=\omega_{2} \kappa_{T} L_{T}+\gamma_{U T} T_{U}-\left(\tau_{2}+\mu+\delta_{d T}\right) T_{D}$

e population of TB recovered individual is increased by the treatment of detected individual (at the rate $\tau_{2}$ ) this population later decreased by natural death and disease induced death (at a rate $\mu$ and $\delta_{\mathrm{RT}}$ ). Thus $\frac{d R_{T}}{d t}=\tau_{2} T_{D}-\left(\mu+\delta_{R T}\right) R_{T}$

Hence, we have 


$$
\begin{aligned}
& \frac{d S}{d t}=\pi-\lambda_{H} S-\lambda_{T} S-\mu S \\
& \frac{d L_{H}}{d t}=\varepsilon_{1} \lambda_{H} S-K_{1} L_{H} \\
& \frac{d H_{U}}{d t}=\left(1-\varepsilon_{1}\right) \lambda_{H} S+K_{2} L_{H}-K_{3} H_{U} \\
& \frac{d H_{D}}{d t}=\omega_{1} \kappa_{H} L_{H}+\gamma_{U H} H_{U}-K_{4} H_{D} \\
& \frac{d T_{H}}{d t}=\tau_{1} H_{D}-K_{5} T_{H} \\
& \frac{d L_{T}}{d t}=\varepsilon_{2} \lambda_{T} S-K_{6} L_{T} \\
& \frac{d T_{U}}{d t}=\left(1-\varepsilon_{2}\right) \lambda_{T} S+K_{7} L_{T}-K_{8} T_{U} \\
& \frac{d T_{D}}{d t}=\omega_{2} \kappa_{T} L_{T}+\gamma_{U T} T_{U}-K_{9} T_{D} \\
& \frac{d R_{T}}{d t}=\tau_{2} T_{D}-K_{10} R_{T}
\end{aligned}
$$

Where,

$K_{1}=\left(\kappa_{H}+\mu\right), K_{2}=\left(1-\omega_{1}\right) \kappa_{H}, K_{3}=\left(\gamma_{U H}+\mu+\delta_{U H}\right), K_{4}=\left(\tau_{1}+\mu+\delta_{d H}\right)$,

$K_{5}=\left(\mu+\delta_{R H}\right), K_{6}=\left(\kappa_{T}+\mu\right) L_{T}, K_{7}=\left(1-\omega_{2}\right) \kappa_{T} L_{T}, K_{8}=\left(\gamma_{U T}+\mu+\delta_{U T}\right)$,

$K_{9}=\left(\tau_{2}+\mu+\delta_{d T}\right), \quad K_{10}=\left(\mu+\delta_{R T}\right)$

Table 1. Description of parameters and variables

\begin{tabular}{|l|l|}
\hline Parameters & Definitions \\
\hline$L_{H}$ & Susceptible individuals \\
\hline$H_{D}$ & Letected HIV individual \\
\hline$H_{U}$ & Undetected HIV individual \\
\hline$L_{T}$ & Latent TB individual \\
\hline$T_{U}$ & Undetected TB individual \\
\hline$T_{D}$ & Detected TB individual \\
\hline$R_{T}$ & TB recover individual \\
\hline$T_{H}$ & Treated HIV individual \\
\hline$\tau_{1}, \tau_{2}$ & Treatment rate for Detected HIV and TB \\
\hline$\lambda_{H}, \lambda_{T}$ & Effective contact rates for HIV and TB \\
\hline$\pi$ & Recruitment rate into the population \\
\hline$\mu$ & \\
\hline
\end{tabular}




\begin{tabular}{|c|c|}
\hline $1-\varepsilon_{1}$ & Fast progressors for HIV \\
\hline$\varepsilon_{2}$ & Slow progressors for TB \\
\hline $1-\varepsilon_{2}$ & Fast progressors for TB \\
\hline$\kappa_{H}$ & Progression rate of HIV. \\
\hline$\kappa_{T}$ & Progression rate of TB. \\
\hline$\omega_{1}$ & Endogenous reactivation rate of HIV \\
\hline$\omega_{2}$ & Endogenous reactivation rate of TB \\
\hline$\gamma_{U H}$ & Detection rate for undetected HIV \\
\hline$\gamma_{U T}$ & Detection rate for undetected TB \\
\hline$\delta_{U T}, \delta_{d T}, \delta_{R T}$ & $\begin{array}{l}\text { Tuberculosis-induced mortality rate for classes } \\
T_{U}, T_{D}, R \text { respectively }\end{array}$ \\
\hline$\beta_{T}, \beta_{H}$ & Effective contact rate for TB and HIV \\
\hline$\delta_{U H}, \delta_{d H}$ & $\begin{array}{l}\text { HIV-induced mortality rate for classes } \\
H_{U}, H_{D} \text { respectively }\end{array}$ \\
\hline$\eta_{U}, \eta_{d H}, \eta_{T H}$ & $\begin{array}{l}\text { HIV Modification parameters for classes } \\
H_{U}, H_{D}, T_{H} \text { respectively }\end{array}$ \\
\hline$\eta_{U}, \eta_{d T}, \eta_{R T}$ & $\begin{array}{l}\text { TB Modification parameters for classes } \\
T_{U}, T_{D}, R_{T} \text { respectively }\end{array}$ \\
\hline
\end{tabular}

\section{Mathematical analysis}

Lemma 1. The closed set

$D_{1}=\left\{\left(S, L_{H}, \eta_{U} H_{U}, \eta_{d H} H_{D}, \eta_{T H} T_{H}, L_{T}, \eta_{U} T_{U}, \eta_{d H} T_{D}, \eta_{R T} R_{T}\right) \in R^{9}{ }_{+}: N \leq \pi / \mu\right.$ is positively-invariant and attracting with respect to the model (1)

Proof: Consider the biologically-feasible region $D$, defined above. The rate of change of the total population, obtained by adding all equations of the model (13), is given by

$$
\frac{d N}{d t}=\pi-\mu N-\delta_{U H}-\delta_{d H}-\delta_{T H}-\delta_{U T}-\delta_{d T}-\delta_{R T}
$$

It follows that $\frac{d N}{d t}<0$ whenever $N>\frac{\pi}{\mu}$. Furthermore, since $\frac{d N}{d t} \leq \pi-\mu N$, it is clear that $N(t) \leq \frac{\pi}{\mu}$ if $N(0) \leq \frac{\pi}{\mu}$. Therefore, all solutions of the model with initial conditions in $D$ remain in $D$ for all $t>0$ (i.e., the $\omega$-limits sets of the system (13) are contained in $D$ ). Thus, $D$ is positively-invariant and attracting. In this region, the model can be considered as been epidemiologically and mathematically well posed

3.1. Analysis of the sub-models. before analyzing the full model, it is pertinent to gain insights into the dynamics of the models for HIV only and TB only model.

3.1.1. HIV-only model. The HIV only model is given by 
$\frac{d S}{d t}=\pi-\lambda_{H} S-\lambda_{T} S-\mu S$

$\frac{d L_{H}}{d t}=\varepsilon_{1} \lambda_{H} S-K_{1} L_{H}$

$\frac{d H_{U}}{d t}=\left(1-\varepsilon_{1}\right) \lambda_{H} S+K_{2} L_{H}-K_{3} H_{U}$

$\frac{d H_{D}}{d t}=\omega_{1} \kappa_{H} L_{H}+\gamma_{U H} H_{U}-K_{4} H_{D}$

$\frac{d T_{H}}{d t}=\tau_{1} H_{D}-K_{5} T_{H}$

Where,

$\lambda_{H}=\beta_{H} \frac{\left(L_{H}+\eta_{U} H_{U}+\eta_{d H} H_{D}+\eta_{T} T_{H}\right)}{N}$

For this model, it can be shown that the region,

$D_{1}=\left\{\left(S+L_{H}+\eta_{U} H_{U}+\eta_{d H} H_{D}+\eta_{T} T_{H}\right) \in R_{+}^{5}: N \leq \pi / \mu\right.$

\subsection{Stability of the disease free equilibrium (dfe)}

Disease free equilibrium

For critical points, we set

$\frac{d S}{d t}=\frac{d L_{H}}{d t}=\frac{d H_{U}}{d t}=\frac{d H_{D}}{d t}=\frac{d T_{H}}{d t}=0$

At disease free equilibrium, it is assumed that there is no infection, and then we set $\lambda_{H}=0$

So, $L_{H}=0, H_{U}=0, H_{D}=0, T_{H}=0$

Disease free equilibrium of HIV model only is given by

$\varepsilon_{0}=\left\{\frac{\pi}{\mu}, 0,0,0,0\right\}$

\subsection{Derivation of basic reproduction number $\left(r_{0}\right)$ for hiv only}

The basic reproduction number $R_{H}$ of the model () is calculated by using the next generation matrix (Driessche and Watmough, 2002). Using their approach (Driessche and Watmough, 2002), we have,

$$
F i=\left(\begin{array}{l}
F 1 \\
F 2 \\
F 3 \\
F 4
\end{array}\right)=\left(\begin{array}{l}
\varepsilon_{1} \lambda_{H} S \\
\left(1-\varepsilon_{1}\right) \lambda_{H} S \\
0 \\
0
\end{array}\right)
$$

$V i=\left(\begin{array}{l}V 1 \\ V 2 \\ V 3 \\ V 4\end{array}\right)=\left(\begin{array}{l}-K_{1} L_{H} \\ K_{2} L_{H}-K_{3} H_{U} \\ \omega_{1} \kappa_{H} L_{H}+\gamma_{U H} H_{U}-K_{4} H_{D} \\ \tau_{1} H_{D}-K_{5} T_{H}\end{array}\right)$

Where 


$$
\mathrm{F}=\left(\begin{array}{cccc}
\varepsilon_{1} \beta_{H} & \varepsilon_{1} \beta_{H} \eta_{U} & \varepsilon_{1} \beta_{H} \eta_{d H} & \varepsilon_{1} \beta_{H} \eta_{T} \\
\left(1-\varepsilon_{1}\right) \beta_{H} & \left(1-\varepsilon_{1}\right) \beta_{H} \eta_{U} & \left(1-\varepsilon_{1}\right) \beta_{H} \eta_{d H} & \left(1-\varepsilon_{1}\right) \beta_{H} \eta_{T} \\
0 & 0 & 0 & 0 \\
0 & 0 & 0 & 0
\end{array}\right)
$$

and

$$
V=\left(\begin{array}{llll}
K_{1} & 0 & 0 & 0 \\
-K_{2} & K_{3} & 0 & 0 \\
-\omega_{1} \kappa_{H} & -\gamma_{U H} & K_{4} & 0 \\
0 & 0 & -\tau_{1} & K_{5}
\end{array}\right)
$$

The reproduction number is the dominant eigen values of $F \times V^{-1}$. Thus,

$$
R_{H}=\left\{\begin{array}{l}
\frac{1}{K_{4} K_{5} K_{1} K_{3}}\left(\left(\left(\left(K_{4} \eta_{U}+\gamma_{U H} \eta_{d H}\right) K_{5}+\gamma_{U H} \eta_{T} \tau_{1}\right) K_{1}\left(1-\varepsilon_{1}\right)+\right.\right. \\
\left.\left.\left(\left(K_{2} \gamma_{U H} \eta_{d H}+\left(\eta_{d H} \omega_{1} \kappa_{H}+K_{4}\right) K_{3}+K_{4} K_{2} \eta_{U}\right) K_{5}+\eta_{T} \tau_{1}\left(\omega_{1} \kappa_{H} K_{3}+K_{2} \gamma_{U H}\right)\right) \varepsilon_{1}\right) \beta_{H}\right)
\end{array}\right\}
$$

Lemma 2. The DFE of the HIV-only model (15), given by (18), is locally asymptotically stable (LAS) if $R_{H}<1$, and unstable if $R_{H}>1$.

The threshold quantity $R_{H}$ is the basic reproduction number of the normalized model system

(15) for HIV infection in a population. It measures the average number of new secondary infections generated by a single infected individual in his or her infectious period in a susceptible population [1].

\subsection{Local stability of disease free equilibrium (dfe) -hiv only}

Theorem 1.: The disease free equilibrium of the system (3.115) is locally asymptotically stable (LAS) if $R_{H}<1$ and unstable if $R_{H}>1$.

Proof: To determine the local stability of $E_{0}$, the following Jacobian matrix is computed corresponding to equilibrium point $E_{0}$. Considering the stability of the disease free equilibrium at the critical point $(0,0,0,0,0)$. Equation (3.115), we have

$J_{H}=\left(\begin{array}{lllll}-\mu & 0 & 0 & 0 & 0 \\ 0 & -K_{1} & 0 & 0 & 0 \\ 0 & K_{2} & -K_{3} & 0 & 0 \\ 0 & \omega_{1} \kappa_{H} & \gamma_{U H} & -K_{4} & 0 \\ 0 & 0 & 0 & \tau_{1} & -K_{5}\end{array}\right)$

Then the characteristics equations are obtained as $\left|J_{H}-\lambda I\right|=0$ (where I is $5^{\star} 5$ identity matrix).

Where,

$$
\lambda I=\left[\begin{array}{lllll}
\lambda & 0 & 0 & 0 & 0 \\
0 & \lambda & 0 & 0 & 0 \\
0 & 0 & \lambda & 0 & 0 \\
0 & 0 & 0 & \lambda & 0 \\
0 & 0 & 0 & 0 & \lambda
\end{array}\right]
$$


Hence $\left|J_{H}-\lambda I\right|=0$ implies that

$$
\left(\begin{array}{lllll}
-\mu-\lambda_{1} & 0 & 0 & 0 & 0 \\
0 & -K_{1}-\lambda_{2} & 0 & 0 & 0 \\
0 & K_{2} & -K_{3}-\lambda_{3} & 0 & 0 \\
0 & \omega_{1} \kappa_{H} & \gamma_{U H} & -K_{4}-\lambda_{4} & 0 \\
0 & 0 & 0 & \tau_{1} & -K_{5}-\lambda_{5}
\end{array}\right)
$$

From equation (21), clearly $\lambda_{1}=-\mu, \lambda_{2}=-K_{1}, \lambda_{3}=-K_{3}, \lambda_{4}=-K_{4}, \lambda_{5}=-K_{5}$

Hence, the corresponding eigen values of equation (15) are;

$\lambda_{1}=-\mu, \lambda_{2}=-\left(\kappa_{H}+\mu\right), \lambda_{3}=-\left(\gamma_{U H}+\mu+\delta_{U H}\right), \quad \lambda_{4}=-\left(\tau_{1}+\mu+\delta_{U H}\right), \quad \lambda_{5}=-\left(\mu+\delta_{R H}\right)$

Where,

$K_{1}=\left(\kappa_{H}+\mu\right), K_{2}=\left(1-\omega_{1}\right) \kappa_{H}, K_{3}=\left(\gamma_{U H}+\mu+\delta_{U H}\right), \quad K_{4}=\left(\tau_{1}+\mu+\delta_{d H}\right), \quad K_{5}=\left(\mu+\delta_{R H}\right) \operatorname{Sinc}$ e all the real roots are negative, real and distinct. Hence, disease free equilibrium of the HIV only model (15) is locally asymptotically stable (LAS).

\subsection{Global stability of disease free equilibrium (hiv)}

Here, the global asymptotic stability (GAS) property of the DEF of the HIV model only will be explored.

Theorem 2: The disease free-equilibrium of the system (15) is globally asymptotically stable whenever $R_{H}<1$ and unstable if $R_{H}>1$.

Proof: It follows that $S=N^{*}-L_{H}-H_{U}-H_{D}-T_{H}$ at steady state. The proof is based on using the comparison theorem (Lakshmkantham et al, 1989) to prove the global stability. The rate of change of the variables representing the infected component of the system can be written as follows.

$$
\begin{aligned}
& \frac{d L_{H}}{d t}=\varepsilon_{1} \lambda_{H}\left(N^{*}-L_{H}-H_{U}-H_{D}-H_{W}\right)-K_{1} L_{H} \\
& \frac{d H_{U}}{d t}=\left(1-\varepsilon_{1}\right) \lambda_{H}\left(N^{*}-L_{H}-H_{U}-H_{D}-H_{W}\right)-K_{2} L_{H}-K_{3} H_{U} \\
& \frac{d H_{D}}{d t}=\omega_{1} K_{H} L_{H}+\gamma_{U H} H_{U}-K_{4} H_{D} \\
& \frac{d T_{H}}{d t}=\tau_{1} H_{D}-K_{5} T_{H} \\
& \left.D^{*}=\left\{L_{H}, H_{U}, H_{D}, T_{H}\right) \in R_{+}^{4}: L_{H}+H_{U}+H_{D}+T_{H} \leq N^{*}\right\}
\end{aligned}
$$

For the model (15), the associated reproduction number, denoted by $R_{H}$ where

$$
R_{H}=\left\{\begin{array}{l}
\frac{1}{K_{4} K_{5} K_{1} K_{3}}\left(\left(\left(\left(K_{4} \eta_{U}+\gamma_{U H} \eta_{d H}\right) K_{5}+\gamma_{U H} \eta_{T} \tau_{1}\right) K_{1}\left(1-\varepsilon_{1}\right)+\right.\right. \\
\left.\left.\left(\left(K_{2} \gamma_{U H} \eta_{d H}+\left(\eta_{d H} \omega_{1} \kappa_{H}+K_{4}\right) K_{3}+K_{4} K_{2} \eta_{U}\right) K_{5}+\eta_{T} \tau_{1}\left(\omega_{1} \kappa_{H} K_{3}+K_{2} \gamma_{U H}\right)\right) \varepsilon_{1}\right) \beta_{H}\right)
\end{array}\right\}
$$

The DFE of the model (15) is GAS in $\mathrm{D}^{*}$ if $R_{H}<1$.

Using comparison method, we have, 


$$
\left(\begin{array}{l}
\frac{d L_{H}}{d t} \\
\frac{d H_{U}}{d t} \\
\frac{d H_{D}}{d t} \\
\frac{d T_{H}}{d t}
\end{array}\right)=(F-V)\left(\begin{array}{l}
L_{H} \\
H_{U} \\
H_{D} \\
T_{H}
\end{array}\right)-F i\left(\begin{array}{l}
L_{H} \\
H_{U} \\
H_{D} \\
T_{H}
\end{array}\right)
$$

According to (Castillo Chavez et al 2002) and (Driessche and Watmough 2002), all eigenvalues of the matrix (F - V) have negative real parts.

$$
\left(\begin{array}{cccc}
\left(\varepsilon_{1} \beta_{H}-K_{1}\right)-\lambda & \varepsilon_{1} \beta_{H} \eta_{U} & \varepsilon_{1} \beta_{H} \eta_{d H} & \varepsilon_{1} \beta_{H} \eta_{T} \\
\left(1-\varepsilon_{1}\right) \beta_{H}+K_{2} & \left(\left(1-\varepsilon_{1}\right) \beta_{H} \eta_{U}-K_{3}\right)-\lambda & \left(1-\varepsilon_{1}\right) \beta_{H} \eta_{d H} & \left(1-\varepsilon_{1}\right) \beta_{H} \eta_{T} \\
\omega_{1} \kappa_{H} & \gamma_{U H} & -K_{4}-\lambda & 0 \\
0 & 0 & \tau_{1} & -K_{5}-\lambda
\end{array}\right)=0
$$

From equation (23), we have

$$
\left[\begin{array}{l}
\left(\left(\varepsilon_{1} \beta_{H}-K_{1}\right)-\lambda\right)\left(\left(1-\varepsilon_{1}\right) \beta_{H} \eta_{U}-K_{3}-\lambda\right)\left(-K_{4}-\lambda\right)\left(-K_{5}-\lambda\right) \\
-\left(\varepsilon_{1} \beta_{H} \eta_{U}\right)\left(\left(1-\varepsilon_{1}\right) \beta_{H}+K_{2}\right)\left(-K_{4}-\lambda\right)\left(-K_{5}-\lambda\right)+\left(\varepsilon_{1} \beta_{H} \eta_{d H}\right)\left(\left(1-\varepsilon_{1}\right) \beta_{H}+K_{2}\right)\left(\gamma_{U H}\left(-K_{5}-\lambda\right)\right) \\
-\left(\varepsilon_{1} \beta_{H} \eta_{T}\right)\left(\left(1-\varepsilon_{1}\right) \beta_{H}+K_{2}\right)\left(\gamma_{U H}+\tau_{1}\right)
\end{array}\right]=0
$$

Thus, from equation (24), the characteristic equation is given by

$$
\lambda^{4}+a_{4} \lambda^{3}+a_{3} \lambda^{2}+a_{2} \lambda+a_{1}
$$

Where

$$
\begin{aligned}
& a_{4}=\left(\left(\left(\eta_{U}-1\right) \varepsilon_{1}-\eta_{U}\right) \beta_{H}+K_{4} K_{5} K_{1} K_{3}\right) \\
& a_{3}=\left(\left(\left(\left(\eta_{U}-1\right) K_{5}+K_{1} \eta_{U}+\left(\eta_{U}-1\right) K_{4}-\eta_{d H} \omega_{1} \kappa_{H}-K_{2} \eta_{U}+\gamma_{U H} \eta_{d H}-K_{3}\right) \varepsilon_{1}-K_{1} \eta_{U}-K_{4} \eta_{U}\right.\right. \\
& \left.\left.\quad-K_{5} \eta_{U}-\gamma_{U H} \eta_{d H}\right) \beta_{H}+\left(K_{4}+K_{1}+K_{3}\right) K_{5}+\left(K_{4}+K_{3}\right) K_{1}+K_{4} K_{3}\right) \\
& a_{2}=\left(\left(\left(\left(K_{1} \eta_{U}+\left(\eta_{U}-1\right) K_{4}-\eta_{d H} \omega_{1} \kappa_{H}-K_{2} \eta_{U}+\gamma_{U H} \eta_{d H}-K_{3}\right) K_{5}+\left(K_{4} \eta_{U}+\gamma_{U H} \eta_{d H}\right) K_{1}+\left(-K_{2} \eta_{U}-K_{3}\right) K_{4}\right.\right.\right. \\
& \left.+\left(-K_{2} \eta_{d H}++\tau_{1} \eta_{T}\right) \gamma_{U H}-\kappa_{H} \omega_{1}\left(K_{3} \eta_{d H}++\tau_{1} \eta_{T}\right)\right) \varepsilon_{1}+\left(-K_{1} \eta_{U}-K_{4} \eta_{U}-\gamma_{U H} \eta_{d H}\right) K_{5}+\left(-K_{4} \eta_{U}-\gamma_{U H} \eta_{d H}\right) K_{1} \\
& \left.\left.\quad-\gamma_{U H} \eta_{T} \tau_{1}\right) \beta_{H}+\left(\left(K_{4}+K_{3}\right) K_{1}+K_{4} K_{3}\right) K_{5}+K_{1} K_{4} K_{3}\right) \\
& a_{1}=K_{4} K_{5} K_{1} K_{3}-\left(\left(\left(\left(K_{4} \eta_{U}+\gamma_{U H} \eta_{d H}\right) K_{5}+\gamma_{U H} \eta_{T} \tau_{1}\right) K_{1}\left(1-\varepsilon_{1}\right)+\right.\right. \\
& \left.\left.\left(\left(K_{2} \gamma_{U H} \eta_{d H}+\left(\eta_{d H} \omega_{1} \kappa_{H}+K_{4}\right) K_{3}+K_{4} K_{2} \eta_{U}\right) K_{5}+\eta_{T} \tau_{1}\left(\omega_{1} \kappa_{H} K_{3}+K_{2} \gamma_{U H}\right)\right) \varepsilon_{1}\right) \beta_{H}\right)
\end{aligned}
$$

Thus according to Routh Hurwitz Criteria of order four (4),

$n=4: a_{1}>0, a_{3}>0, a_{4}>0$, and $a_{1} a_{2} a_{3}>a_{3}^{2}+a_{1}^{2} a_{4}$.

Then for $a_{1}>0$ we have

$$
\begin{aligned}
& \left(\left(\left(\left(K_{4} \eta_{U}+\gamma_{U H} \eta_{d H}\right) K_{5}+\gamma_{U H} \eta_{T} \tau_{1}\right) K_{1}\left(1-\varepsilon_{1}\right)+\right.\right. \\
& \frac{\left.\left.\left(\left(K_{2} \gamma_{U H} \eta_{d H}+\left(\eta_{d H} \omega_{1} \kappa_{H}+K_{4}\right) K_{3}+K_{4} K_{2} \eta_{U}\right) K_{5}+\eta_{T} \tau_{1}\left(\omega_{1} \kappa_{H} K_{3}+K_{2} \gamma_{U H}\right)\right) \varepsilon_{1}\right) \beta_{H}\right)}{K_{4} K_{5} K_{1} K_{3}}<1,
\end{aligned}
$$


Implying that

$R_{H}<1$

According to (Castillo-Chavez et al. 2002) and (Driessche and Watmough 2002), all eigenvalues of the matrix $F-V$ have negative real parts. It follows that the linearized differential inequality above is stable whenever $R_{H}<1$. Consequently $S=\left(L=H_{U}=H_{D}=H_{W}=0\right) \rightarrow(0,0,0,0)$ at $t \quad \rightarrow \infty$. Substituting $L=H_{U}=H_{D}=H_{W}=0$ in $\left(R_{H}\right)$ gives $S(t) \rightarrow S(0)$ as $t \rightarrow \infty$. Hence, we have established that the disease free equilibrium is globally asymptotically stable whenever $R_{H}<1$ and unstable $R_{H}>1$.

\subsection{Hiv sensitivity analysis}

It is useful to conduct an investigation to determine how sensitive the threshold quantity basic reproduction number is with respect to its parameters, this will help us to know which of the parameters causes most reduction in $R_{o}$ and parameters that have high impact on $R_{o}$ and these should be targeted by intervention strategies in order to have most effective control of the disease. This analysis tells us how crucial and important each parameter is to disease transmission. We compute the normalized forward sensitivity index of the reproduction number with respect to its parameters.

Definition: If a variable ' $c$ ' depends differentiably on a parameter ' $w$ ', then, the normalized forward sensitivity index of 'c' with respect to ' $w$ ' is denoted by $\mathrm{Xc}$, which is defined as $X_{c}=\frac{c}{w} \frac{\partial w}{\partial c}$

As we have explicit formula for $R_{o}$, we derive an analytical expression for the sensitive of $R_{o}$ as

$$
X_{w}^{R_{o}}=\frac{d R_{o}}{d w} \times \frac{w}{R_{o}}
$$

Sensitivity analysis for each parameter involved in $R_{H}$, is therefore calculated and show in the table below.

Table 2. VALUES OF NUMERICAL SENSITIVITY OF HIV

\begin{tabular}{|c|l|}
\hline PARAMETER & SENSITIVITY VALUE \\
\hline$\tau_{1}$ & 0.0000000 \\
\hline$\mu$ & -0.0777526 \\
\hline$\delta_{d H}$ & -0.0061828 \\
\hline$\delta_{U H}$ & -0.0080448 \\
\hline$\gamma_{U H}$ & 0.0000000 \\
\hline$\delta_{T H}$ & -0.0106258 \\
\hline$\varepsilon_{1}$ & 0.9685616 \\
\hline$\beta_{H}$ & 1.000000 \\
\hline$\kappa_{H}$ & -0.9431861 \\
\hline
\end{tabular}

3.7. TB -only model. The TB only model is given by 


$$
\begin{aligned}
& \frac{d S}{d t}=\pi-\lambda_{T} S-\mu S \\
& \frac{d L_{T}}{d t}=\varepsilon_{2} \lambda_{T} S-K_{6} L_{T} \\
& \frac{d T_{U}}{d t}=\left(1-\varepsilon_{2}\right) \lambda_{T} S+K_{7} L_{T}-K_{8} T_{U} \\
& \frac{d T_{D}}{d t}=\omega_{2} \kappa_{T} L_{T}+\gamma_{U T} T_{U}-K_{9} T_{D} \\
& \frac{d R_{T}}{d t}=\tau_{2} T_{D}-K_{10} T_{D}
\end{aligned}
$$

Where,

$$
\lambda_{T}=\beta_{T} \frac{\left(L_{T}+\eta_{U} T_{U}+\eta_{d T} T_{D}+\eta_{R T} R_{T}\right)}{N}
$$

For this model, it can be shown that the region,

$D_{1}=\left\{\left(S+L_{T}+\eta_{U} T_{U}+\eta_{d T} T_{D}+\eta_{R T} R_{T}\right) \in R_{+}^{5}: N \leq \pi / \mu\right.$

\subsection{Stability of the disease free equilibrium (dfe)}

Disease free equilibrium

For critical points, we set

$\frac{d S}{d t}=\frac{d L_{T}}{d t}=\frac{d T_{U}}{d t}=\frac{d T_{D}}{d t}=\frac{d R_{T}}{d t}=0$

At disease free equilibrium, it is assumed that there is no infection, and then we set $\lambda_{T}=0$

So, $L_{T}=0, T_{U}=0, T_{D}=0, R_{T}=0$

Disease free equilibrium of TB model only is given by

$\varepsilon_{0}=\left\{\frac{\pi}{\mu}, 0,0,0,0\right\}$

\subsection{Derivation of basic reproduction number $\left(R_{0}\right)$ for TB-only}

the basic reproduction number $R_{T}$ of the model (26) is calculated by using the next generation matrix (driessche and watmough, 2002). using their approach (driessche and watmough, 2002), we have,

$$
\begin{aligned}
F i=\left(\begin{array}{l}
F 1 \\
F 2 \\
F 3 \\
F 4
\end{array}\right) & =\left(\begin{array}{l}
\varepsilon_{2} \lambda_{T} S \\
\left(1-\varepsilon_{2}\right) \lambda_{T} S \\
0 \\
0
\end{array}\right) \\
V i & =\left(\begin{array}{l}
V 1 \\
V 2 \\
V 3 \\
V 4
\end{array}\right)=\left(\begin{array}{l}
-K_{6} L_{T} \\
K_{7} L_{T}-K_{8} T_{U} \\
\omega_{2} \kappa_{T} L_{T}+\gamma_{U T} T_{U}-K_{9} T_{D} \\
\tau_{2} T_{D}-K_{10} T_{D}
\end{array}\right)
\end{aligned}
$$


where

$$
\mathrm{F}=\left(\begin{array}{cccc}
\varepsilon_{2} \beta_{T} & \varepsilon_{2} \beta_{T} \eta_{U} & \varepsilon_{2} \beta_{H} \eta_{d T} & \varepsilon_{2} \beta_{T} \eta_{R T} \\
\left(1-\varepsilon_{2}\right) \beta_{T} & \left(1-\varepsilon_{2}\right) \beta_{T} \eta_{U} & \left(1-\varepsilon_{2}\right) \beta_{T} \eta_{d T} & \left(1-\varepsilon_{1}\right) \beta_{T} \eta_{R T} \\
0 & 0 & 0 & 0 \\
0 & 0 & 0 & 0
\end{array}\right)
$$

and

$$
V=\left(\begin{array}{llll}
K_{6} & 0 & 0 & 0 \\
-K_{7} & K_{8} & 0 & 0 \\
-\omega_{2} \kappa_{T} & -\gamma_{U T} & K_{9} & 0 \\
0 & 0 & -\tau_{2} & K_{10}
\end{array}\right)
$$

the reproduction number is the dominant eigen values of $F \times V^{-1}$. thus,

$$
R_{T}=\left\{\begin{array}{l}
\frac{1}{K_{9} K_{10} K_{6} K_{8}}\left(\left(\left(\left(K_{9} \eta_{U}+\gamma_{U T} \eta_{d T}\right) K_{10}+\gamma_{U T} \eta_{R T} \tau_{2}\right) K_{6}\left(1-\varepsilon_{2}\right)+\right.\right. \\
\left.\left.\left(\left(K_{7} \gamma_{U T} \eta_{d T}+\left(\eta_{d T} \omega_{2} \kappa_{T}+K_{9}\right) K_{8}+K_{9} K_{7} \eta_{U}\right) K_{10}+\eta_{R T} \tau_{2}\left(\omega_{2} \kappa_{T} K_{8}+K_{7} \gamma_{U T}\right)\right) \varepsilon_{2}\right) \beta_{T}\right)
\end{array}\right\}
$$

lemma 3. the dfe of the tb-only model (26), given by (30), is locally asymptotically stable (las) if $R_{T}<1$, and unstable if $R_{T}>1$.

the threshold quantity $R_{T}$ is the basic reproduction number of the normalized model system

(26) for tb infection in a population. it measures the average number of new secondary infections generated by a single infected individual in his or her infectious period in a susceptible

population [1].

\subsection{Local stability of disease free equilibrium (dfe) TB-only}

Theorem 3.: The disease free equilibrium of the system (26) is locally asymptotically stable (LAS) if $R_{T}<1$ and unstable if $R_{T}>1$.

Proof: To determine the local stability of $E_{0}$, the following Jacobian matrix is computed corresponding to equilibrium point $E_{0}$. Considering the stability of the disease free equilibrium at the critical point $(0,0,0,0,0)$. Equation 26$)$, we have

$$
J_{T}=\left(\begin{array}{lllll}
-\mu & 0 & 0 & 0 & 0 \\
0 & -K_{6} & 0 & 0 & 0 \\
0 & K_{7} & -K_{8} & 0 & 0 \\
0 & \omega_{2} \kappa_{T} & \gamma_{U T} & -K_{9} & 0 \\
0 & 0 & 0 & \tau_{2} & -K_{10}
\end{array}\right)
$$

Then the characteristics equations are obtained as $\left|J_{T}-\lambda I\right|=0$ (where I is $5^{\star} 5$ identity matrix).

Where, 


$$
\lambda I=\left[\begin{array}{lllll}
\lambda & 0 & 0 & 0 & 0 \\
0 & \lambda & 0 & 0 & 0 \\
0 & 0 & \lambda & 0 & 0 \\
0 & 0 & 0 & \lambda & 0 \\
0 & 0 & 0 & 0 & \lambda
\end{array}\right]
$$

Hence $\left|J_{H}-\lambda I\right|=0$ implies that

$$
\left(\begin{array}{lllll}
-\mu-\lambda_{1} & 0 & 0 & 0 & 0 \\
0 & -K_{6}-\lambda_{2} & 0 & 0 & 0 \\
0 & K_{7} & -K_{8}-\lambda_{3} & 0 & 0 \\
0 & \omega_{2} \kappa_{T} & \gamma_{U T} & -K_{9}-\lambda_{4} & 0 \\
0 & 0 & 0 & \tau_{2} & -K_{10}-\lambda_{5}
\end{array}\right)
$$

From equation (32), clearly $\lambda_{1}=-\mu, \lambda_{2}=-K_{6}, \lambda_{3}=-K_{8}, \lambda_{4}=-K_{9}, \quad \lambda_{5}=-K_{10}$

Hence, the corresponding eigen values of equation (26) are;

$\lambda_{1}=-\mu, \lambda_{2}=-\left(\kappa_{T}+\mu\right), \lambda_{3}=-\left(\gamma_{U T}+\mu+\delta_{U T}\right), \quad \lambda_{4}=-\left(\tau_{1}+\mu+\delta_{U H}\right), \quad \lambda_{5}=-\left(\mu+\delta_{R H}\right)$

Where,

$K_{6}=\left(\kappa_{T}+\mu\right), K_{7}=\left(1-\omega_{2}\right) \kappa_{T}, K_{8}=\left(\gamma_{U T}+\mu+\delta_{U T}\right), \quad K_{9}=\left(\tau_{2}+\mu+\delta_{d d T}\right), \quad K_{10}=\left(\mu+\delta_{R T}\right) \operatorname{Sin}$ ce all the real roots are negative, real and distinct. Hence, disease free equilibrium of the TB only model (26) is locally asymptotically stable (LAS).

\subsection{Global stability of disease free equilibrium (TB)}

Here, the global asymptotic stability (GAS) property of the DEF of the TB model only will be explored.

Theorem 4: The disease free-equilibrium of the system (26) is globally asymptotically stable whenever $R_{T}<1$ and unstable if $R_{T}>1$.

Proof: It follows that $S=N^{*}-L_{T}-T_{U}-T_{D}-R_{T}$ at steady state. The proof is based on using the comparison theorem (Lakshmkantham et al, 1989) to prove the global stability. The rate of change of the variables representing the infected component of the system can be written as follows.

$$
\begin{aligned}
& \frac{d L_{T}}{d t}=\varepsilon_{2} \lambda_{T}\left(N^{*}-L_{T}-T_{U}-T_{D}-H \backslash R_{T}\right)-K_{6} L_{T} \\
& \frac{d T_{U}}{d t}=\left(1-\varepsilon_{2}\right) \lambda_{T}\left(N^{*}-L_{T}-T_{U}-T_{D}-R_{T}\right)-K_{7} L_{T}-K_{8} T_{U} \\
& \frac{d T_{D}}{d t}=\omega_{2} \kappa_{T} L_{T}+\gamma_{U T} T_{U}-K_{9} T_{D} \\
& \frac{d R_{T}}{d t}=\tau_{2} T_{D}-K_{10} R_{T} \\
& \left.D^{*}=\left\{L_{T}, T_{U}, T_{D}, R_{T}\right) \in R_{+}^{4}: L_{T}+T_{U}+T_{D}+R_{T} \leq N^{*}\right\}
\end{aligned}
$$

For the model (26), the associated reproduction number, denoted by $R_{T}$ where

$$
R_{T}=\left\{\begin{array}{l}
\frac{1}{K_{9} K_{10} K_{6} K_{8}}\left(\left(\left(\left(K_{9} \eta_{U}+\gamma_{U T} \eta_{d T}\right) K_{10}+\gamma_{U T} \eta_{R T} \tau_{2}\right) K_{6}\left(1-\varepsilon_{2}\right)+\right.\right. \\
\left.\left.\left(\left(K_{7} \gamma_{U T} \eta_{d T}+\left(\eta_{d T} \omega_{2} \kappa_{T}+K_{9}\right) K_{8}+K_{9} K_{7} \eta_{U}\right) K_{10}+\eta_{R T} \tau_{2}\left(\omega_{2} \kappa_{T} K_{8}+K_{7} \gamma_{U T}\right)\right) \varepsilon_{2}\right) \beta_{T}\right)
\end{array}\right\}
$$


The DFE of the model (26) given by disease free (), is GAS in $\mathrm{D}^{*}$ if $R_{T}<1$.

Using comparison method, we have,

$\left(\begin{array}{l}\frac{d L_{H}}{d t} \\ \frac{d H_{U}}{d t} \\ \frac{d H_{D}}{d t} \\ \frac{d T_{H}}{d t}\end{array}\right)=(F-V)\left(\begin{array}{l}L_{H} \\ H_{U} \\ H_{D} \\ T_{H}\end{array}\right)-F i\left(\begin{array}{l}L_{H} \\ H_{U} \\ H_{D} \\ T_{H}\end{array}\right)$

According to (Castillo Chavez et al 2002) and (Driessche and Watmough 2002), all eigenvalues of the matrix (F - V) have negative real parts.

$$
\left(\begin{array}{cccc}
\left(\varepsilon_{1} \beta_{H}-K_{1}\right)-\lambda & \varepsilon_{1} \beta_{H} \eta_{U} & \varepsilon_{1} \beta_{H} \eta_{d H} & \varepsilon_{1} \beta_{H} \eta_{T} \\
\left(1-\varepsilon_{1}\right) \beta_{H}+K_{2} & \left(\left(1-\varepsilon_{1}\right) \beta_{H} \eta_{U}-K_{3}\right)-\lambda & \left(1-\varepsilon_{1}\right) \beta_{H} \eta_{d H} & \left(1-\varepsilon_{1}\right) \beta_{H} \eta_{T} \\
\omega_{1} \kappa_{H} & \gamma_{U H} & -K_{4}-\lambda & 0 \\
0 & 0 & \tau_{1} & -K_{5}-\lambda
\end{array}\right)=0
$$

From equation (3.153), we have

$\left[\begin{array}{l}\left(\left(\varepsilon_{2} \beta_{T}-K_{6}\right)-\lambda\right)\left(\left(1-\varepsilon_{2}\right) \beta_{T} \eta_{U}-K_{8}-\lambda\right)\left(-K_{9}-\lambda\right)\left(-K_{10}-\lambda\right) \\ -\left(\varepsilon_{2} \beta_{T} \eta_{U}\right)\left(\left(1-\varepsilon_{2}\right) \beta_{T}+K_{7}\right)\left(-K_{9}-\lambda\right)\left(-K_{10}-\lambda\right)+\left(\varepsilon_{2} \beta_{T} \eta_{d T}\right)\left(\left(1-\varepsilon_{2}\right) \beta_{T}+K_{7}\right)\left(\gamma_{U T}\left(-K_{10}-\lambda\right)\right) \\ -\left(\varepsilon_{2} \beta_{T} \eta_{R T}\right)\left(\left(1-\varepsilon_{2}\right) \beta_{T}+K_{7}\right)\left(\gamma_{U T}+\tau_{2}\right)\end{array}\right]=0$

Thus, from equation (35), the characteristic equation is given by

$\lambda^{4}+b_{4} \lambda^{3}+b_{3} \lambda^{2}+b_{2} \lambda+b_{1}$

Where

$$
\begin{aligned}
& b_{4}=\left(\left(\left(\eta_{U}-1\right) \varepsilon_{2}-\eta_{U}\right) \beta_{t}+K_{9} K_{10} K_{6} K_{8}\right) \\
& b_{3}=\left(\left(\left(\left(\eta_{U}-1\right) K_{10}+K_{6} \eta_{U}+\left(\eta_{U}-1\right) K_{9}-\eta_{d T} \omega_{2} \kappa_{T}-K_{7} \eta_{U}+\gamma_{U T} \eta_{d T}-K_{8}\right) \varepsilon_{2}-K_{6} \eta_{U}-K_{9} \eta_{U}\right.\right. \\
& \left.\left.\quad-K_{10} \eta_{U}-\gamma_{U T} \eta_{d T}\right) \beta_{T}+\left(K_{9}+K_{6}+K_{8}\right) K_{10}+\left(K_{9}+K_{8}\right) K_{6}+K_{9} K_{8}\right)
\end{aligned}
$$$$
b_{2}=\left(\left(\left(\left(K_{6} \eta_{U}+\left(\eta_{U}-1\right) K_{9}-\eta_{d T} \omega_{2} \kappa_{T}-K_{7} \eta_{U}+\gamma_{U T} \eta_{d T}-K_{8}\right) K_{10}+\left(K_{9} \eta_{U}+\gamma_{U T} \eta_{d T}\right) K_{6}+\left(-K_{7} \eta_{U}-K_{8}\right) K_{9}\right.\right.\right.
$$$$
\left.+\left(-K_{7} \eta_{d T}++\tau_{2} \eta_{R T}\right) \gamma_{U T}-\kappa_{T} \omega_{2}\left(K_{8} \eta_{d T}++\tau_{2} \eta_{R T}\right)\right) \varepsilon_{2}+\left(-K_{6} \eta_{U}-K_{9} \eta_{U}-\gamma_{U T} \eta_{d T}\right) K_{10}+\left(-K_{9} \eta_{U}-\gamma_{U T} \eta_{d T}\right) K_{6}
$$$$
\left.\left.-\gamma_{U T} \eta_{R T} \tau_{2}\right) \beta_{T}+\left(\left(K_{9}+K_{8}\right) K_{6}+K_{9} K_{8}\right) K_{10}+K_{6} K_{9} K_{8}\right)
$$$$
b_{1}=K_{9} K_{10} K_{6} K_{8}-\left(\left(\left(\left(K_{9} \eta_{U}+\gamma_{U T} \eta_{d T}\right) K_{10}+\gamma_{U T} \eta_{R T} \tau_{2}\right) K_{6}\left(1-\varepsilon_{2}\right)+\right.\right.
$$$$
\left.\left.\left(\left(K_{7} \gamma_{U T} \eta_{d T}+\left(\eta_{d T} \omega_{2} \kappa_{T}+K_{9}\right) K_{8}+K_{9} K_{7} \eta_{U}\right) K_{10}+\eta_{R T} \tau_{2}\left(\omega_{2} \kappa_{T} K_{8}+K_{7} \gamma_{U T}\right)\right) \varepsilon_{2}\right) \beta_{T}\right)
$$

Thus according to Routh Hurwitz Criteria of order four (4),

$n=4: b_{1}>0, b_{3}>0, b_{4}>0$, and $b_{1} b_{2} b_{3}>b_{3}^{2}+b_{1}^{2} b_{4}$.

Then for $b_{1}>0$ we have

$$
\begin{aligned}
& \left(\left(\left(\left(K_{9} \eta_{U}+\gamma_{U T} \eta_{d T}\right) K_{10}+\gamma_{U T} \eta_{R T} \tau_{2}\right) K_{6}\left(1-\varepsilon_{2}\right)+\right.\right. \\
& \frac{\left.\left.\left(\left(K_{7} \gamma_{U T} \eta_{d T}+\left(\eta_{d T} \omega_{2} \kappa_{T}+K_{9}\right) K_{8}+K_{9} K_{7} \eta_{U}\right) K_{10}+\eta_{R T} \tau_{2}\left(\omega_{2} \kappa_{T} K_{8}+K_{7} \gamma_{U T}\right)\right) \varepsilon_{2}\right) \beta_{T}\right)}{K_{9} K_{10} K_{6} K_{8}}<1
\end{aligned}
$$


Implying that

$R_{T}<1$

According to (Castillo-Chavez et al. 2002) and (Driessche and Watmough 2002), all eigenvalues of the matrix $F-V$ have negative real parts. It follows that the linearized differential inequality above is stable whenever $R_{T}<1$. Consequently $S=\left(L_{T}=T_{U}=T_{D}=R_{T}=0\right) \rightarrow(0,0,0,0)$ at $t \quad \rightarrow \infty$. Substituting $L_{T}=T_{U}=T_{D}=R_{T}=0$ in $\left(R_{T}\right)$ gives $S(t) \rightarrow S(0)$ as $t \rightarrow \infty$. Hence, we have established that the disease free equilibrium is globally asymptotically stable whenever $R_{T}<1$ and unstable $R_{T}>1$.

\subsection{TB Sensitivity analysis}

Also to each parameters involved in $R_{T}$, the sensitivity indices of $R_{T}$ with respect to each parameter is calculated below, Results obtained were tabulated below as follows:

Table 3. Values of numerical sensitivity of tb

\begin{tabular}{|l|l|}
\hline PARAMETERS & SENSITIVITY VALUES \\
\hline$\beta_{T}$ & 0.9999999 \\
\hline$\gamma_{U T}$ & -0.0000000 \\
\hline$\mu$ & -0.0598660 \\
\hline$\varepsilon_{2}$ & 0.9575027 \\
\hline & -0.0064931 \\
\hline$\delta_{U T}$ & -0.0110215 \\
\hline$\delta_{R T}$ & -0.0165677 \\
\hline$\kappa_{T}$ & -1.0089064 \\
\hline$\tau_{2}$ & $-4^{\star} 10^{-10}$ \\
\hline
\end{tabular}

\subsection{Analysis of the full model}

Consider now the Co-infection model (14) of HIV-TB

Disease free equilibrium of the full model is given by

$\varepsilon_{1}=\left(S^{*}, L_{H}{ }^{*}, H_{U}{ }^{*}, H_{D}{ }^{*}, T_{H}{ }^{*}, L_{T}{ }^{*}, T_{U}{ }^{*}, T_{D}{ }^{*}, R_{T}{ }^{*}\right)$

$\varepsilon_{1}=\left(\frac{\pi}{\mu}, 0,0,0,0,0,0,0,0\right)$

\subsection{Derivation of basic reproduction number $\left(\mathbf{R}_{0}\right)$ for HIV-TB co-infection}

\section{The Next Generation Matrix $\left(F . V^{-1}\right)$ Method}

The basic reproduction number of the model (16) $R_{T H}$ is calculated by using the next generation matrix (Driessche and Watmough, 2002). Using their approach (Driessche and Watmough, 2002).

The associated matrices $\mathrm{F}$ and $\mathrm{V}$, are given, respectively, 


$$
F=\left(\begin{array}{lcccccccc}
\varepsilon_{1} \beta_{H} & \varepsilon_{1} \beta_{H} \eta_{U} & \varepsilon_{1} \beta_{H} \eta_{d H} & \varepsilon_{1} \beta_{H} \eta_{T} & 0 & 0 & 0 & 0 & 0 \\
\left(1-\varepsilon_{1}\right) \beta_{H} & \left(1-\varepsilon_{1}\right) \beta_{H} \eta_{U} & \left(1-\varepsilon_{1}\right) \beta_{H} \eta_{d H} & \left(1-\varepsilon_{1}\right) \beta_{H} \eta_{T} & 0 & 0 & 0 & 0 & 0 \\
0 & 0 & 0 & 0 & 0 & 0 & 0 & 0 & 0 \\
0 & 0 & 0 & 0 & 0 & 0 & 0 & 0 & 0 \\
\varepsilon_{2} \beta_{T} & \varepsilon_{2} \beta_{T} \eta_{U} & \varepsilon_{2} \beta_{T} \eta_{d T} & \varepsilon_{2} \beta_{T} \eta_{R T} & 0 & 0 & 0 & 0 & 0 \\
\left(1-\varepsilon_{2}\right) \beta_{T} & \left(1-\varepsilon_{2}\right) \beta_{T} \eta_{U} & \left(1-\varepsilon_{2}\right) \beta_{T} \eta_{d T} & \left(1-\varepsilon_{2}\right) \beta_{T} \eta_{R T} & 0 & 0 & 0 & 0 & 0 \\
0 & 0 & 0 & 0 & 0 & 0 & 0 & 0 & 0 \\
0 & 0 & 0 & 0 & 0 & 0 & 0 & 0 & 0
\end{array}\right)
$$

and

$V=\left(\begin{array}{lllll}K_{1} & 0 & 0 & 0 & 0 \\ -K_{2} & K_{3} & 0 & 0 & 0 \\ -\omega_{1} \kappa_{H} & -\gamma_{U H} & K_{4} & 0 & 0 \\ 0 & 0 & -\tau_{1} & K_{5} & 0 \\ K_{6} & 0 & 0 & 0 & 0 \\ -K_{7} & K_{8} & 0 & 0 & 0 \\ -\omega_{2} \kappa_{T} & -\gamma_{U T} & K_{9} & 0 & 0 \\ 0 & 0 & -\tau_{2} & K_{10} & 0\end{array}\right.$

$\left.\begin{array}{lll}0 & 0 & 0 \\ 0 & 0 & 0 \\ 0 & 0 & 0 \\ 0 & 0 & 0 \\ 0 & 0 & 0 \\ 0 & 0 & 0 \\ 0 & 0 & 0 \\ 0 & 0 & 0\end{array}\right)$

The reproduction number is the dominant eigen values of $F \times V^{-1}$. Thus,

$$
R=\left\{\begin{array}{l}
\frac{1}{K_{4} K_{5} K_{1} K_{3} K_{9} K_{10} K_{6} K_{8}}\left(\left(\left(-K_{8} K_{9} \varepsilon_{2} \beta_{H}\left(K_{4} \eta_{U}+\gamma_{U H} \eta_{d H}\right) K_{1}^{2}+\left(\left(\left(-\beta_{H}\left(-1+\varepsilon_{1}\right)\left(K_{4} \eta_{U}+\gamma_{U H} \eta_{d H}\right) K_{8}\right.\right.\right.\right.\right.\right. \\
\left.\left.-K_{3} K_{4} \eta_{U} \beta_{T}\left(-1+\varepsilon_{2}\right)\right) K_{6}+K_{3} K_{4} \varepsilon_{2} \beta_{T}\left(K_{7} \eta_{U}+K_{8}\right)\right) K_{9}-K_{4} \eta_{d T}\left(\gamma_{U H}\left(-1+\varepsilon_{2}\right) K_{6}-\right. \\
\left.\left.\left.\varepsilon_{2}\left(K_{8} \kappa_{T} \omega_{2}+K_{7} \gamma_{U T}\right)\right) K_{3} \beta_{T}\right) K_{1}+K_{9} K_{6}\left(\left(K_{2} \eta_{U}+K_{3}\right) K_{4}+\eta_{d H}\left(K_{3} \kappa_{H} \omega_{1}+K_{2} \gamma_{U H}\right)\right) K_{8} \varepsilon_{1} \beta_{H}\right) K_{5} \\
\left.-\left(K_{1}^{2} \gamma_{U H} \varepsilon_{2}+K_{6} \gamma_{U H}\left(-1+\varepsilon_{1}\right) K_{1}-K_{6} \varepsilon_{1}\left(K_{3} \kappa_{H} \omega_{1}+K_{2} \gamma_{U H}\right)\right) K_{9} K_{8} \eta_{T H} \beta_{H} \tau_{1}\right) K_{10}-K_{4} \eta_{R T} \tau_{2} \\
\left(\gamma_{U T}\left(-1+\varepsilon_{2}\right) K_{6}-\varepsilon_{2}\left(K_{8} \kappa_{T} \omega_{2}+K_{7} \gamma_{U T}\right)\right) K_{3} K_{1} K_{5} \beta_{T}
\end{array}\right\}(35)
$$

\subsection{Local stability of disease free equilibrium (dfe) of HIV-TB model}

Theorem 5: The disease free equilibrium of the system (16) is locally asymptotically stable (LAS) if $R_{H T}<1$ and unstable if $R_{H T}>1$.

Proof: Thus, to determine the local stability of $E_{0}$, the following matrix is computed corresponding to equilibrium point $E_{0}$. considering the stability of the disease free equilibrium at the critical point $(0,0,0,0,0,0,0,0,0)$. Then we have,

The jacobian matrix of equation (16) is 


$$
J_{H T}=\left(\begin{array}{lllllllll}
-\mu & 0 & 0 & 0 & 0 & 0 & 0 & 0 & 0 \\
0 & -K_{1} & 0 & 0 & 0 & 0 & 0 & 0 & 0 \\
0 & K_{2} & -K_{3} & 0 & 0 & 0 & 0 & 0 & 0 \\
0 & \omega_{1} \kappa_{H} & \gamma_{U H} & -K_{4} & 0 & 0 & 0 & 0 & 0 \\
0 & 0 & 0 & \tau 1 & -K_{5} & 0 & 0 & 0 & 0 \\
0 & 0 & 0 & 0 & 0 & -K_{6} & 0 & 0 & 0 \\
0 & 0 & 0 & 0 & 0 & K_{7} & -K_{8} & 0 & 0 \\
0 & 0 & 0 & 0 & 0 & \kappa_{T} \omega_{2} & \gamma_{U T} & -K_{9} & 0 \\
0 & 0 & 0 & 0 & 0 & 0 & 0 & \tau_{2} & -K_{10}
\end{array}\right)
$$

hen the characteristics equation is obtained as $\left|J_{H T}-\lambda I\right|=0$ (where I is 9*9 identity matrix). Where,

$$
I=\left[\begin{array}{lllllllll}
\lambda & 0 & 0 & 0 & 0 & 0 & 0 & 0 & 0 \\
0 & \lambda & 0 & 0 & 0 & 0 & 0 & 0 & 0 \\
0 & 0 & \lambda & 0 & 0 & 0 & 0 & 0 & 0 \\
0 & 0 & 0 & \lambda & 0 & 0 & 0 & 0 & 0 \\
0 & 0 & 0 & 0 & \lambda & 0 & 0 & 0 & 0 \\
0 & 0 & 0 & 0 & 0 & \lambda & 0 & 0 & 0 \\
0 & 0 & 0 & 0 & 0 & 0 & \lambda & 0 & 0 \\
0 & 0 & 0 & 0 & 0 & 0 & 0 & \lambda & 0 \\
0 & 0 & 0 & 0 & 0 & 0 & 0 & 0 & \lambda
\end{array}\right]
$$

Hence $\left|J_{H T}-\lambda I\right|=0$ implies that

$$
\left(\begin{array}{llllllllll}
-\mu-\lambda_{1} & 0 & 0 & 0 & 0 & 0 & 0 & 0 & 0 \\
0 & -K_{1}-\lambda_{2} & 0 & 0 & 0 & 0 & 0 & 0 & 0 \\
0 & K_{2} & -K_{3}-\lambda_{3} & 0 & 0 & 0 & 0 & 0 & 0 \\
0 & \omega_{1} \kappa_{H} & \gamma_{U H} & -K_{4}-\lambda_{4} & 0 & 0 & 0 & 0 & 0 \\
0 & 0 & 0 & \tau 1 & -K_{5}-\lambda_{5} & 0 & 0 & 0 & 0 \\
0 & 0 & 0 & 0 & 0 & -K_{6}-\lambda_{6} & 0 & 0 & 0 \\
0 & 0 & 0 & 0 & 0 & K_{7} & -K_{8}-\lambda_{7} & 0 & 0 \\
0 & 0 & 0 & 0 & 0 & \kappa_{T} \omega_{2} & \gamma_{U T} & -K_{9}-\lambda_{8} & 0 \\
0 & 0 & 0 & 0 & 0 & 0 & 0 & \tau_{2} & -K_{10}-\lambda_{9}
\end{array}\right)
$$


From equation (37), we have

$$
\begin{aligned}
& \lambda_{1}=-\mu, \lambda_{2}=-K_{1}, \lambda_{3}=-K_{3}, \lambda_{4}=-K_{4}, \lambda_{5}=-K_{5}, \lambda_{6}=-K_{6}, \lambda_{7}=-K_{7}, \lambda_{8}=-K_{9} \\
& \lambda_{9}=-K_{10}
\end{aligned}
$$

Since all the real roots are negative, real and distinct. Hence, disease free equilibrium of the HIV-TB model (16) is locally asymptotically stable (LAS).

\subsection{Global stability of disease free equilibrium (HIV-TB)}

Theorem 6: The disease free-equilibrium of the system (16) is globally asymptotically stable whenever $R_{H T}<1$ and unstable if $R_{H T}>1$.

Proof: It follows that at steady state. The proof is based on using the comparison theorem (Lakshmkantham et al, 1989), to prove the global stability.

The DFE of the model (1) given by disease free, is GAS in $\mathrm{D}^{*}$ if $R_{H T}<1$.

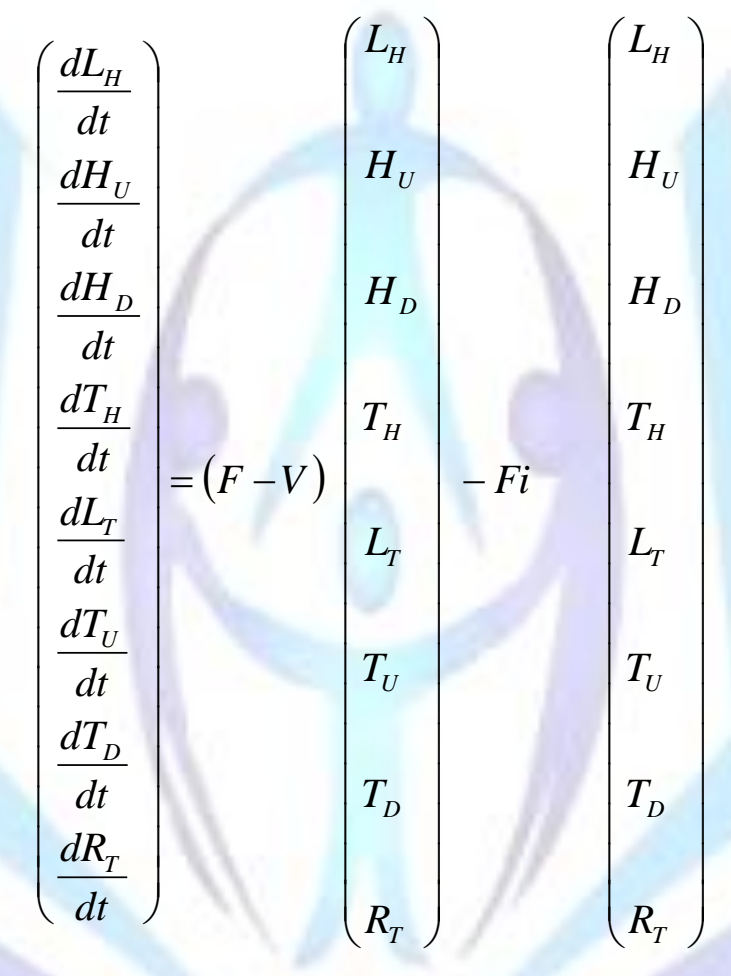

Where

$$
F=\left(\begin{array}{lcccccccc}
\varepsilon_{1} \beta_{H} & \varepsilon_{1} \beta_{H} \eta_{U} & \varepsilon_{1} \beta_{H} \eta_{d H} & \varepsilon_{1} \beta_{H} \eta_{T} & 0 & 0 & 0 & 0 & 0 \\
\left(1-\varepsilon_{1}\right) \beta_{H} & \left(1-\varepsilon_{1}\right) \beta_{H} \eta_{U} & \left(1-\varepsilon_{1}\right) \beta_{H} \eta_{d H} & \left(1-\varepsilon_{1}\right) \beta_{H} \eta_{T} & 0 & 0 & 0 & 0 & 0 \\
0 & 0 & 0 & 0 & 0 & 0 & 0 & 0 & 0 \\
0 & 0 & 0 & 0 & 0 & 0 & 0 & 0 & 0 \\
\varepsilon_{2} \beta_{T} & \varepsilon_{2} \beta_{T} \eta_{U} & \varepsilon_{2} \beta_{T} \eta_{d T} & \varepsilon_{2} \beta_{T} \eta_{R T} & 0 & 0 & 0 & 0 & 0 \\
\left(1-\varepsilon_{2}\right) \beta_{T} & \left(1-\varepsilon_{2}\right) \beta_{T} \eta_{U} & \left(1-\varepsilon_{2}\right) \beta_{T} \eta_{d T} & \left(1-\varepsilon_{2}\right) \beta_{T} \eta_{R T} & 0 & 0 & 0 & 0 & 0 \\
0 & 0 & 0 & 0 & 0 & 0 & 0 & 0 & 0 \\
0 & 0 & 0 & 0 & 0 & 0 & 0 & 0 & 0
\end{array}\right)
$$

and 


$V=\left(\begin{array}{llllllll}K_{1} & 0 & 0 & 0 & 0 & 0 & 0 & 0 \\ -K_{2} & K_{3} & 0 & 0 & 0 & 0 & 0 & 0 \\ -\omega_{1} \kappa_{H} & -\gamma_{U H} & K_{4} & 0 & 0 & 0 & 0 & 0 \\ 0 & 0 & -\tau_{1} & K_{5} & 0 & 0 & 0 & 0 \\ K_{6} & 0 & 0 & 0 & 0 & 0 & 0 & 0 \\ -K_{7} & K_{8} & 0 & 0 & 0 & 0 & 0 & 0 \\ -\omega_{2} \kappa_{T} & -\gamma_{U T} & K_{9} & 0 & 0 & 0 & 0 & 0 \\ 0 & 0 & -\tau_{2} & K_{10} & 0 & 0 & 0 & 0\end{array}\right)$

According to (Castillo-Chavez et al. 2002) and (Driessche and Watmough 2002), all eigenvalues of the matrix $F-V$ have negative real parts. It follows that the linearized differential inequality above is stable whenever $R_{H T}<1$.

Consequently,

$S=\left(L_{H}=H_{U}=H_{D}=H_{W}=L_{T H}=A_{T H}=L_{T H}=L_{H T}=A_{H T}=L_{T}=T_{U}=T_{D}=F_{T}=R_{T}=R_{T H}=0\right) \rightarrow$ $(0,0,0,0,0)$ at $t \rightarrow \infty$

Hence, we have established that the disease free equilibrium is globally asymptotically stable whenever $R_{H T}<1$ and unstable $R_{H T}>1$.

\subsection{HIV-TB Sensitivity analysis}

Also to each parameters involved in $R_{T H}$, the sensitivity indices of $R_{T H}$ with respect to each parameter is calculated below,

Results obtained were tabulated below as follows:

Table 4. Values of numerical sensitivity of HIV/TB

\begin{tabular}{|l|l|}
\hline \multicolumn{1}{|c|}{ PARAMETERS } & SENSITIVITY VALUES \\
\hline$\beta_{T}$ & 0.6005990 \\
\hline$\beta_{H}$ & 0.3994010 \\
\hline$\kappa_{H}$ & -0.9588586 \\
\hline$\omega_{2}$ & $5.8991105 * 10^{-13}$ \\
\hline$\varepsilon_{1}$ & 0.3931723 \\
\hline$\kappa_{T}$ & -0.5379916 \\
\hline$\gamma_{U H}$ & -0.000567 \\
\hline$\gamma_{U T}$ & $-3.0628670 * 10^{-10}$ \\
\hline$\mu$ & -0.0665580 \\
\hline$\varepsilon_{2}$ & 0.5685420 \\
\hline$\delta_{U H}$ & -0.0012241 \\
\hline$\delta_{T H}$ & -0.0141147 \\
\hline$\delta_{d H}$ & -0.0012580 \\
\hline$\delta_{U T}$ & -0.0066195 \\
\hline
\end{tabular}




\begin{tabular}{|l|l|}
\hline$\delta_{d T}$ & -0.0038995 \\
\hline$\delta_{R T}$ & -0.0099506 \\
\hline$\tau_{1}$ & -0.0476514 \\
\hline$\tau_{2}$ & -0.0057460 \\
\hline
\end{tabular}

4.0. Numerical simulation. The full model is simulated to access the impact of each parameter in the dynamical spread of HIV-TB co-infection.

Table 5. Parameter Values used in Numerical Simulations

\begin{tabular}{lcc}
\multicolumn{1}{c}{ Parameters } & Value & Sources \\
$\mu$ & 2000 & Okuonghae etal., (2007) \\
$\tau_{1}, \tau_{2}$ & 0.02 & Becerra et al., (2005) \\
$\varepsilon_{1}, \varepsilon_{2}$ & $0.20619,0.30619$ & Assumed \\
$\kappa_{H}$ & $0.7,0.7$ & Sharomi et al., (2008) \\
$\kappa_{T}$ & 0.2522 & Daniel et al., (2007) \\
$\omega_{1}, \omega_{2}$ & 0.3522 & Assumed \\
$\gamma_{U H}, \gamma_{U T}$ & $0.2,0.2$ & Sharomi et al., (2008) \\
$\beta_{H} \beta_{T}$ & $0.2,0.3$ & Assumed \\
$\delta_{U H}, \delta_{d H}$ & $0.1,0.2$ & Colijn et al., (2007) \\
$\eta_{U}, \eta_{d H}, \eta_{T}$ & $0.3,0.1$ & Colijn et al., (2007) \\
$\delta_{U T}, \delta_{d T}, \delta_{R T}$ & $0.001,0.001,0.001$ & Sharomi et al., (2008) \\
$\eta_{d T}, \eta_{R T}$ & $0.3,0.1,0.1$ & Daniel \& Andrei(2007)
\end{tabular}

Graph of total population when $\beta_{H}=0.3$

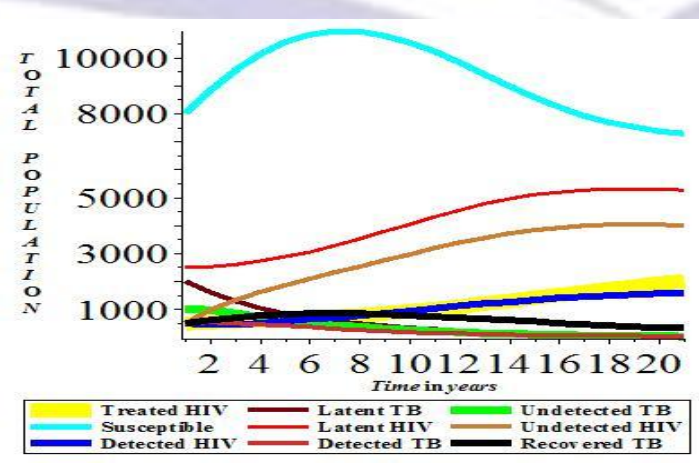

Fig. 1.
Graph of total population when $\beta_{H}=0.6$

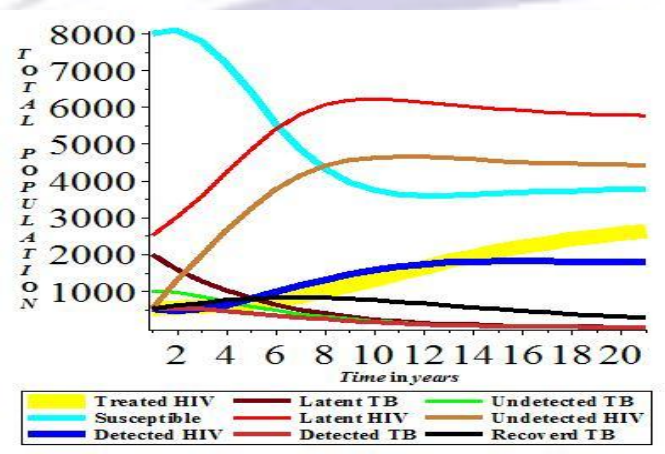

Fig. 2.

Graph of total population when $\beta_{H}=0.9$

Graph of total population when $\beta_{T}=0.3$ 




Fig.3.

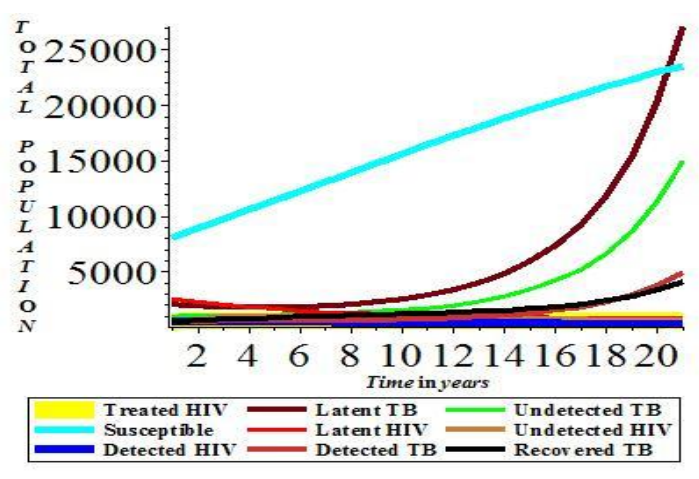

Fig. 4.
Graph of total population when $\beta_{T}=0.6$

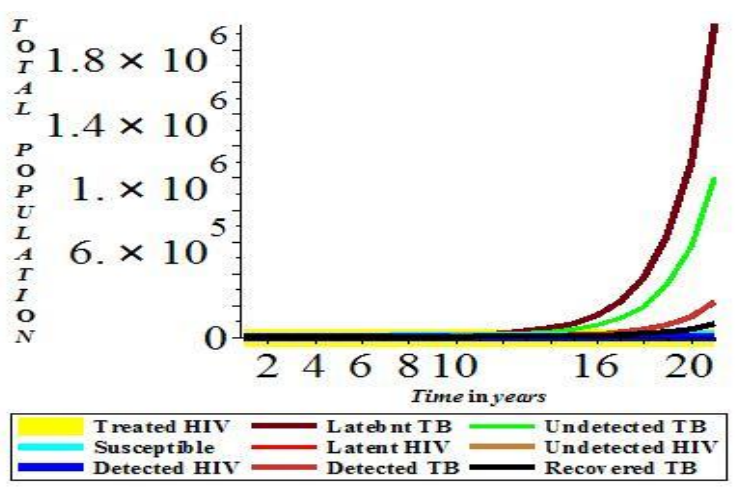

Fig. 5.
Graph of total population when $\beta_{T}=0.9$



Fig. 6.

Graph of total population when $\varepsilon_{1}=0.2 \quad$ Graph of total population when $\varepsilon_{1}=0.6$

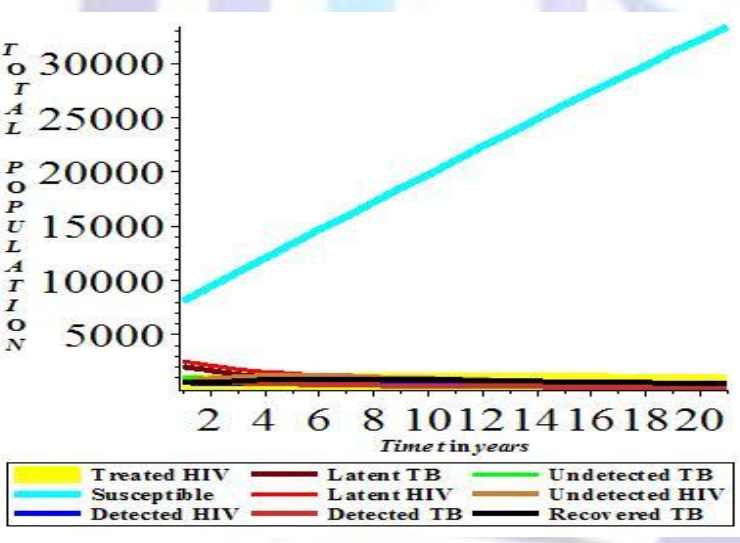

Fig. 7.

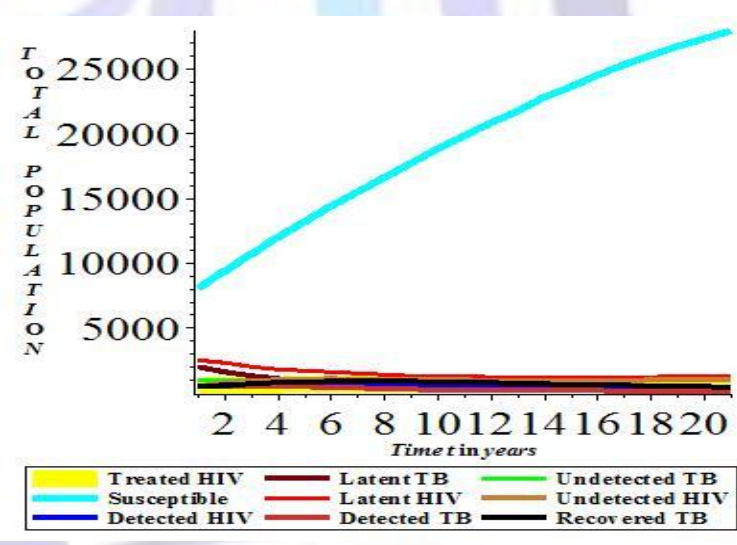

Fig. 8. 


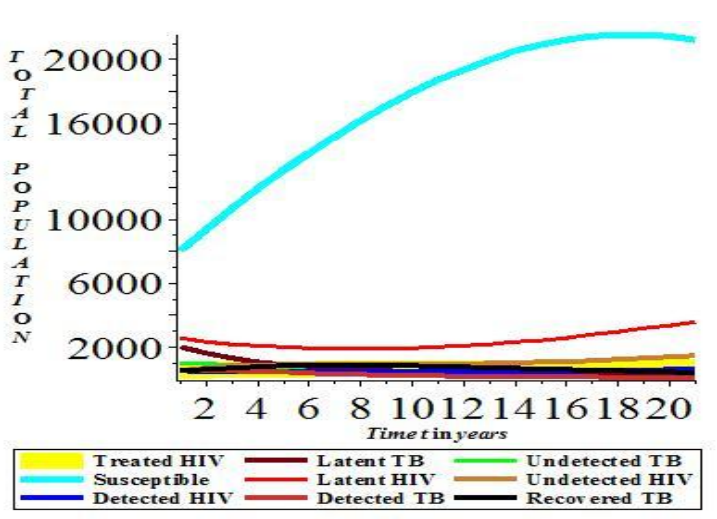

Fig. 9.

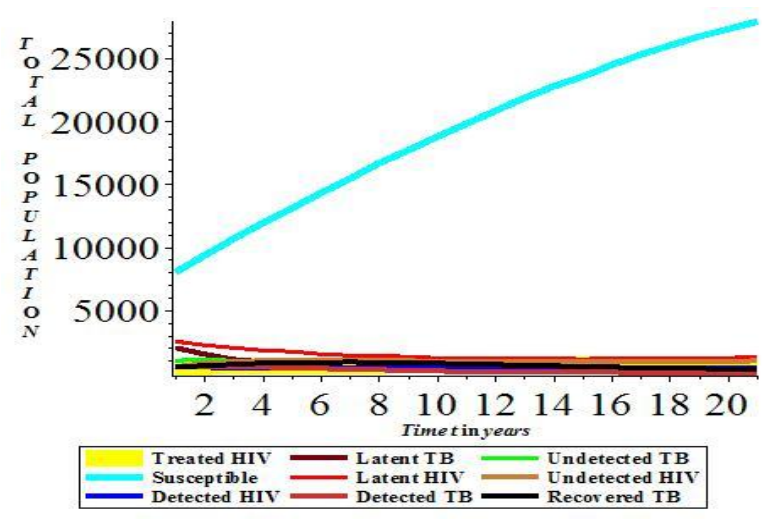

Fig. 10.
Graph of total population when $\varepsilon_{2}=0.7$

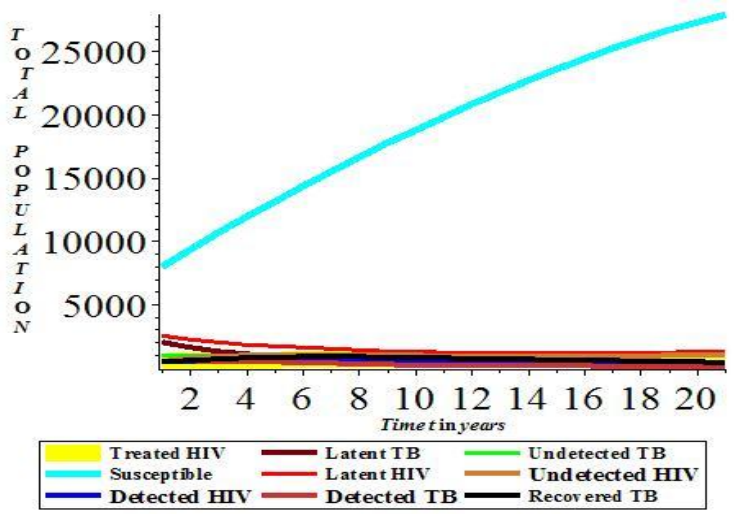

Fig. 11.

Graph of total population when $\gamma_{U H}=0.4$

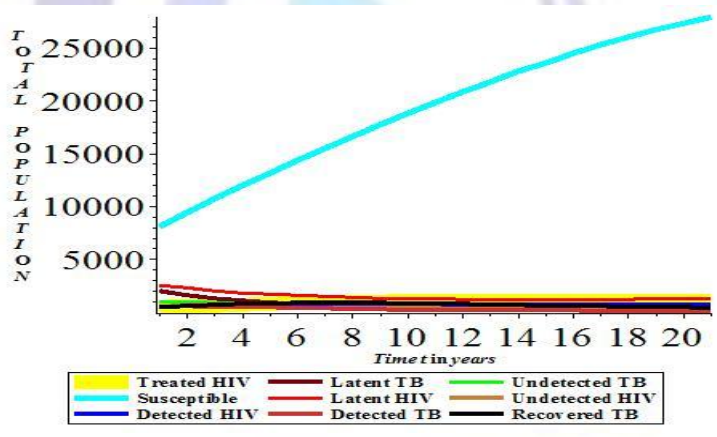

Fig. 13.
Graph of total population when $\varepsilon_{2}=0.9$

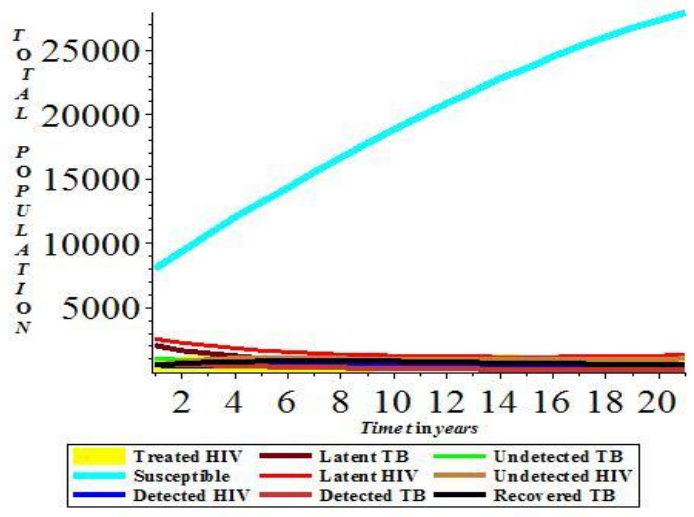

Fig. 12.

Graph of total population when $\gamma_{U H}=0.7$

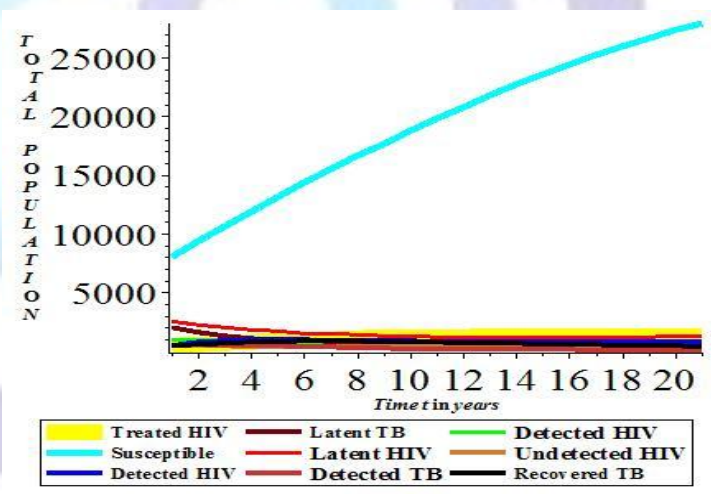

Fig. 14.

Graph of total population when $\gamma_{U H}=0.9 \quad$ Graph of total population when $\gamma_{U T}=0.4$ 


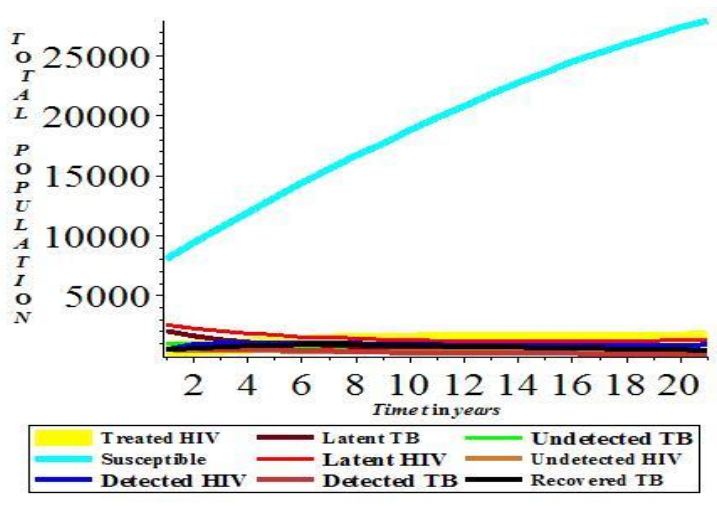

Fig. 15.



Fig. 16.

\section{Graph of total population when $\gamma_{U T}=0.7 \quad$ Graph of total population when $\gamma_{U T}=0.9$}

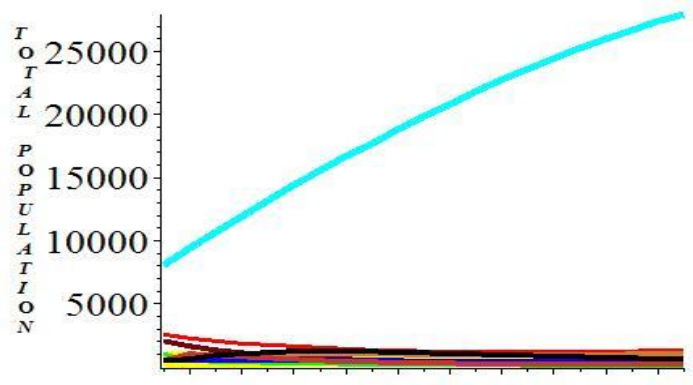

$2 \quad 4 \quad 6 \quad 8 \quad 101214161820$

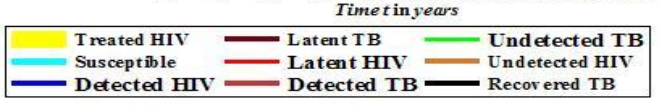

Fig.17.

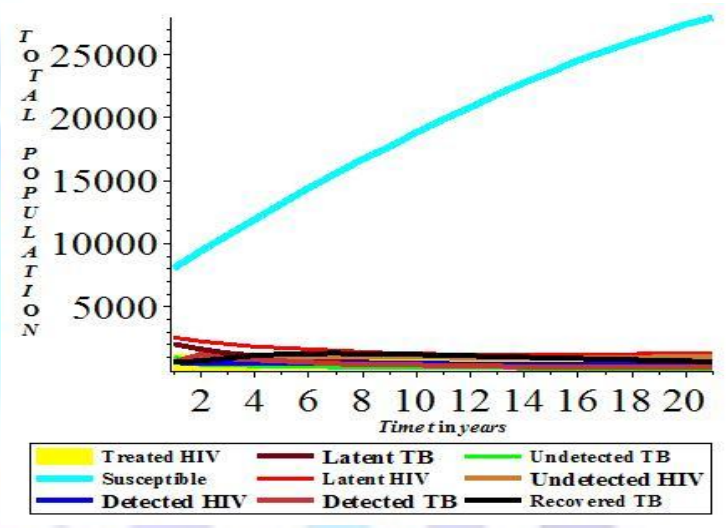

Fig. 18.

\subsection{Discussion of results}

We analyzed a HIV-TB mathematical model, by evaluating the sensitivity indices of parameters involved in the basic reproduction number to know the parameters that need more attention. It is used to discover parameters that have a high impact on basic reproduction number and should be targeted by intervention strategies.

It is observed from the table 2, 3 and 4 . that when the parameters with positive sensitivity values increase while the remaining parameters remain constant, the value of basic reproduction number increases, and this increase the endemicity of the disease in the population. The increment in basic reproduction number makes it more difficult to have disease free environment Parameters with negative sensitivity values have less effect in the dynamical spread of HIV-TB co-infection.

Transmission rate $\beta_{H}, \beta_{T}$ and Fast progressor $\varepsilon_{1}, \varepsilon_{2}$ are the most sensitive parameters in the analysis. Medical practitioners need to work on positive indices parameters most especially parameters that have pronounced effect on the basic reproduction number $\left(R_{0}\right)$ i.e. transmission rates $\beta_{H}$ and $\beta_{T}$ in order to have stable free disease environment.

Using the data in table 5.1, fig. $1,2,3,4,5$ and 6 shows that effective contact rate $\beta_{H}$ and $\beta_{T}$ decreases the susceptible individuals and increases the latent, infected undetected and infected detected individuals. Fig 7, 8, 9, 10, 11 and 12 shows the effect of progressor on latent and infected undetected individuals. When $\varepsilon_{1}$ increases from 0.2 to 0.9 , it increases latent infected individuals from 2122 to 2297 . However, when progressor of TB increases from 0.2 to 0.9 , it increases TB latent infected individuals from 1502 to 1640. Fig. 13, 14, 15, 16, 17 and 18 shows that detection of infected undetected individuals has a pronounced effect on infected detected and undetected individuals. When the detection of HIV infected undetected individuals increases from 0.4 to 0.9 , it reduces infected undetected individuals from 608 to 369 but increases infected detected individuals from 594 to 832 . Also, when the detection of TB infected undetected individuals increases from 0.4 to 0.9 it reduces infected undetected individuals from 767 to 302 but increases infected detected individuals from 706 to 1166 .

\subsection{Conclusion}

In this paper, a deterministic model for the dynamical transmission of HIV-TB co-infection is presented. The objective is to determine the parameters that influence the dynamical spread of the diseases; the model includes Nine (9) compartmental differential equations. 
A threshold parameter $R_{0}$ is defined and is shown that the disease will spread only if its value exceeds unity, i.e. $R_{0}>1$.

Sensitivity analysis of basic reproduction number $R_{0}$ to all the parameters tell us how crucial and important each parameter is to the disease spread in the environment. It showed that parameters representing the transmission rate of HIV and TB have a large impact on the dynamics of the diseases, and which should be targeted by medical practitioners to forestall the spread of the diseases. Also, fast progressor rate of HIV and TB needs to be checked, effort should be put in place medically to improve immunity of susceptible individuals, from the mathematical analysis, we discovered that people with weak immunity are more prone to the two diseases. It also has great significant effect when the two diseases co-exist in a particular patient.

Numerical simulation shows effects of parameters on the dynamical spread of HIV-TB co-infection and this tells us that a change in these parameters can significantly reduce the spread of HIV-TB co-infection disease in the population over time.

\section{REFERENCES}

1. Adewale, S. O. Podder, C. N. and Gumel, A. B. (2009). Mathematical analysis of a Tuberculosis Transmission Model with DOTS. Canadian Applied Mathematics quarterly volume 17, number 1, spring 2009

2. Aparicio J.P., Capurro A.F. and Castillo-Chavez C. (2002) Markers of disease evolution. The case of Tuberculosis Theoretical Biology 215 , 227-237.

3. Bacaer N., Ouitki R., Pretorius C., Wood R. and Williams B., (2008) Modeling the joint epidemics of TB and HIV in a South African township, Journal of Mathematical Biology $57,557-593$.

4. Becerra M. C., Pachao-Torreblanca I. F., Bayona J., Celi R., Shin S. S., Kim J. Y.,Farmer P. E. and Murray M. (2005). Expanding tuberculosis case detection by screening household contacts, Public Health Report. 120.

5. Chaisson R.E. and Martinson N.A. (2008) Tuberculosis on Africa combating an HIV driven crisis. N Eng J Med, 358.1089-1092.

6. Cohen T., Lipsitch M., Walensky R.P. and Murray M. (2006) Beneficial and perverse effects of Isoniazid preventive therapy for latent tuberculosis Infection in HIV-TB Co-infected populations, proceedings of the National Academy of Science of the United States of America 103, 7042-7047

7. Colijn C., Cohen T. and Murray M. (2007). Emergent heterogeneity in declining tuberculosis epidemics. J. Theor. Biol. 247, 765-774.

8. Curran A, Falcó V, Pahissa A, Ribera E (2012) Management of tuberculosis in HIV-infected patients.AIDS 14:231-246. PMID: 23258298

9. Daniel O. and Andrei K. (2007). Dynamics of tuberculosis: The effect of Direct Observation Therapy Strategy (DOTS) in Nigeria. Mathematical Modelling of Natural Phenomena. 2(1):101-113.

10. Fuzzi, et al. (1996) Journal of Biological mathematics, 37, p 371-375

11. Getahun H, Gunneberg C, Granich R, Nunn P (2010) HIV infection-associated tuberculosis: the epidemiology and the response. Clin Infect Dis Suppl: 3:S201- 207.

12. Gray JM, Cohn DL (2013) Tuberculosis and HIV co-infection. Semin Respir Crit Care Med. 34:32-43.doi: 10.1055/s-0032-1333469 PMID: 23460004

13. M. A Mikucki. Sensitivity analysis of the basic reproduction number and other quantities for infectious disease models. M.Sc. thesis, Colorado State University, 2012.

14. Marison L. (2001) The global epidemiology of HIV/AIDS, British Medical Bulletin 58, 7-18

15. Nita H. S and Jyoti G.(2014) :Modelling of HIV-TB Co-infection Transmission Dynamics. American Journal of Epidemiology and Infectious Disease, 2014, Vol. 2, No. 1, 1-7.

16. Okuonghae D. and Korobeinikov A. (2007). Dynamics of tuberculosis: The effect of Direct Observation Therapy Strategy (DOTS) in Nigeria, Math. Modelling Natural Phenomena 2(1), 101-113.

17. Quinn T.C., (1996) Global burden of the HIV pandemic, Lancet 348, 99-106

18. Sanchez M.S., Lloyd-Smith J.O., Williams B.G., Porco T.C., Ryan S.J., Borgdorff M.W., Mansoer J., Dye C. and Getz W.M. (2009) Incongruent HIV and TB Co-dynamics other, Epidemics 1, 14-20.

in Kenya. Interacting epidemics monitor each

19. Sharomi O., Podder C.N., Gumel A.B., and Song B. (2008). Mathematical analysis of the transmission dynamics of HIV/TB co-infection in the presence of treatment.Mathematical Biosciences and Engineering.5(1):145-174. 
20. Smart T.,(2008) Think TB in people with HIV: The l's for TB control in people with HIV, Nam, London.

21. Theurer C.P., Hopewell P.C., Etias D., Schecter G.F., Rutherford G.W. and Chaisson R.E., (1990) HIV infection in TB patients, Journal of infectious Diseases 162 , 8- 12.

22. West R.W. and Thompson J.R. (1997).Modelling the impact of HIV on the spread of tuberculosis in the United States. Mathematical Biosciences. 143: 35-60.

23. World Health Organization, Global TB control: surveillance Planning financing Geneva, 2006: WHO/HTM/TB/2006.362.

24. World Health Organization (WHO), Tuberculosis Facts, http://www.who.int/tb/publications/2007/factsheet_2007.pdf,

25. World Health Organization. World Health Organization Global Tuberculosis report 2013. Available:http://www.who.int/tb/publications/global_report/en/. Accessed July 20, 2014. 